\title{
Structure of Poly(\(Igamma\)-Benzyl-L-Glutamate) Monolayers at the Gas-Water Interface: A Brewster Angle Microscopy and X-Ray Scattering Study
}

\section{Citation}

Fukumo, Masafumi, Ralf K. Heilmann, Peter S. Pershan, Seungju M. Yu, Jennifer A. Griffiths, and David A. Tirrell. 1999. Structure of poly(\(Igamma)-benzyl-L-glutamate) monolayers at the gaswater interface: A Brewster angle microscopy and $\mathrm{x}$-ray scattering study. Journal of Chemical Physics 111(21): 9761-9777.

\section{Published Version}

doi: $10.1063 / 1.480312$

\section{Permanent link}

http://nrs.harvard.edu/urn-3:HUL.InstRepos:10356598

\section{Terms of Use}

This article was downloaded from Harvard University's DASH repository, and is made available under the terms and conditions applicable to Other Posted Material, as set forth at http:// nrs.harvard.edu/urn-3:HUL.InstRepos:dash.current.terms-of-use\#LAA

\section{Share Your Story}

The Harvard community has made this article openly available.

Please share how this access benefits you. Submit a story.

\section{Accessibility}




\title{
Structure of poly ( $\gamma$-benzyl-L-glutamate) monolayers at the gas-water interface: A Brewster angle microscopy and $x$-ray scattering study
}

\author{
Masafumi Fukuto, Ralf K. Heilmann, and Peter S. Pershan \\ Department of Physics and Division of Engineering and Applied Sciences, Harvard University, Cambridge, \\ Massachusetts 02138 \\ Seungju M. Yu, ${ }^{\text {a) }}$ Jennifer A. Griffiths, ${ }^{\text {b) }}$ and David A. Tirrell ${ }^{\mathrm{c})}$ \\ Polymer Science and Engineering Department, University of Massachusetts, Amherst, Massachusetts 01003
}

(Received 14 June 1999; accepted 9 September 1999)

\begin{abstract}
This paper reports Brewster angle microscopy (BAM), X-ray specular reflectivity (XR), grazing incidence diffraction (GID) and off-specular diffuse scattering (XOSDS) measurements of Langmuir monolayers formed on water by both mono- and polydisperse samples of $\alpha$-helical poly $(\gamma$-benzyl L-glutamate) (PBLG) as a function of area/monomer $A$. The microscopic behavior does not exhibit any discernible effects due to differing dispersity. At low surface densities $(A$ $>\sim 21 \AA^{2} /$ monomer, surface pressure $\Pi=0$ ), BAM images reveal partial surface coverage by solidlike monolayer islands. GID measurements show an interhelix peak corresponding to a local parallel alignment of rodlike PBLG molecules, indicating their tendency to aggregate laterally without external pressure. Compression to $A<21 \AA^{2} /$ monomer first leads to full and uniform surface coverage by the monolayer, followed by a steep rise in $\Pi$ that is accompanied by a decrease in the interhelix distance. Further compression results in a plateau of constant $\Pi$ in the $\Pi-A$ isotherm $\left(\sim 11.5<A<\sim 18.5 \AA^{2} /\right.$ monomer, $\left.\Pi \sim 9 \mathrm{dyn} / \mathrm{cm}\right)$, which has previously been attributed to a first-order monolayer-bilayer transition. The interfacial electron density profiles determined by the XR measurements on both sides of the coexistence plateau provide direct evidence for this transition. On the basis of $\mathrm{X}$-ray scattering results, the film on the high-density side of the plateau is shown to consist of a newly formed incomplete and incommensurate second layer that sits on top of and has lower average density than a homogeneous first layer. GID measurements indicate that the second layer can be characterized by larger interhelix $d$-spacing than the first layer, while XOSDS measurements on the bilayer suggest that the second layer is microscopically inhomogeneous. For both mono- and bilayers, the analysis of observed GID peak widths indicates that the extent of lateral positional correlations between parallel PBLG rods ranges from a few to no more than $\sim 15$ interhelix distances, implying short-range order. (C) 1999 American Institute of Physics. [S0021-9606(99)71345-0]
\end{abstract}

\section{INTRODUCTION}

Langmuir monolayers (LMs) provide one example of real physical systems in which an experimental study of twodimensional (2D) physics should be possible. ${ }^{1-3}$ Macroscopic physical chemistry of LMs has been studied for the better part of this century; however, it is only within the most recent $10-15$ years that the availability of synchrotron generated x-rays made it possible to directly characterize their microscopic structures. ${ }^{4,5}$ A considerable number of synchrotron x-ray scattering experiments have now been conducted on LMs, especially those formed by simple surfactant molecules like fatty acids, fatty alcohols, and phospholipids.

Grazing incidence $\mathrm{x}$-ray diffraction (GID) $)^{4,5}$ studies demonstrated that these "long chain" amphiphilic molecules form close packed $2 \mathrm{D}$ crystalline and liquid crystalline struc-

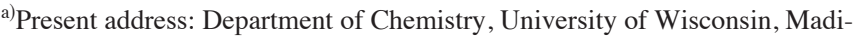
son, Wisconsin 53706.

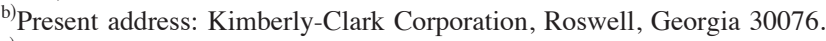

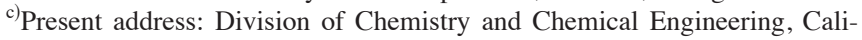
fornia Institute of Technology, Pasadena, California 91125.
}

tures on water in which the chains are oriented either normal to the surface or at relatively small angles to the surface normal. Compression- and temperature-induced phase transformations between ordered LM phases involve changes in the amplitude and direction of the chain tilt. ${ }^{2,5}$ By and large the different 2D crystalline structures observed in the LMs of simple long chain molecules are similar to the various tilted and untilted phases of both thin freely suspended liquid crystal films and bulk smectic liquid crystals. ${ }^{2,6,7}$ One unfortunate aspect of all of these studies is that the number of electrons contained in such simple amphiphilic molecules is often too small to produce measurable scattering from their noncrystalline 2D phases. ${ }^{8,9}$ As a result the elucidation of microscopic structures through $\mathrm{x}$-ray scattering was mostly limited to ordered phases for these LM systems.

This paper describes optical and x-ray scattering studies of LMs formed by polypeptides in the $\alpha$-helical conformation. ${ }^{10-26}$ These rigid rodlike molecules lie down flat on the water surface with $2 \mathrm{D}$ nematiclike structures. As such they differ from the aforementioned more conventional amphiphilic LMs and liquid crystal films in that the director 
$\mathbf{n}$, or principal axis of the molecules, lies within the monolayer plane rather than pointing away from it. Another important difference is that these polypeptide molecules are larger than the simpler amphiphilic molecules studied previously. As a result, the constructive interference in the GID patterns that can be used to characterize the intermolecular packing occurs at smaller angles with larger amplitudes. The combination of this and the one to two orders of magnitude greater number of electrons per molecule has the important consequence that broad GID peaks can be observed from disordered LM phases. For example, a recent GID experiment on a LM formed by $C_{60}$-propylamine adduct molecules ${ }^{27}$ demonstrated that a quantitative measurement of a liquidlike 2D structure factor due to short-range positional correlations is possible when the number of electrons per scattering unit (i.e., molecule) is sufficiently large. The hope is that as a result of the increased scattering power provided by large molecules, it might be possible to characterize the structural changes in the noncrystalline part of $2 \mathrm{D}$ phase diagrams.

Another potential advantage for studies using $\alpha$-helical polypeptides is that without compromising the rigidity of the helical backbones, their intermolecular interactions within the LM plane may be controlled by chemically altering the side chain groups and through copolymerization of different peptide monomer units. Such chemical modifications have already been shown to influence the formation of 3D liquid crystalline phases. ${ }^{28-33}$

The interest in LMs of $\alpha$-helical polypeptides also arises from the fact that many of them can be transferred onto solid substrates as multilayers using the Langmuir-Blodgett (LB) technique. In recent years, various $\alpha$-helical poly(Lglutamates) have been studied both as LMs on water $^{23,25,26,34-37}$ and as LB films on solid substrates..$^{26,34,37-49}$ In most of these studies the primary objective has been to achieve good-quality LB films that possess technologically important properties, ${ }^{50}$ such as lateral homogeneity, ${ }^{23,45}$ thermal $^{37,44,47,49}$ and mechanical ${ }^{45,48}$ stability, low defect density, ${ }^{38,45}$ and optical anisotropy; ${ }^{38,39,42}$ in addition, potential applications, ${ }^{50,51}$ for example, as optical wave guides, ${ }^{38,41,42}$ optical data storage media, ${ }^{43,44}$ and photoresists, ${ }^{37}$ have also been suggested. However, despite the high activity in this research area, direct structural characterizations at the intermolecular level have been limited mostly to transferred LB films, as opposed to their LM counterparts, partly due to experimental difficulties involved with studying liquid surfaces. ${ }^{23}$ As Motschmann et al. ${ }^{23}$ pointed out, the elucidation of LM structures as the state immediately prior to LB-deposition is an important step that may provide additional insights on controlling LB multilayer structures.

We report here the results of Brewster angle microscopy (BAM) and extensive x-ray scattering studies conducted in situ on LMs formed by both monodisperse and polydisperse poly ( $\gamma$-benzyl L-glutamate) (PBLG), ${ }^{12,13,18,25}$ emphasizing the changes in their microscopic structures with lateral compression. The principal results are as follows: (i) Direct microscopic characterization of the first-order monolayerbilayer transition. This transition was originally inferred from the presence of a plateau region of constant $\Pi$ in the surface pressure $(\Pi)$ vs area/monomer $(A)$ isotherms of the PBLG LM. ${ }^{11,12,18}$ Further indirect evidence was provided by Takenaka et al., ${ }^{18}$ who LB-deposited the same number of PBLG layers onto solid substrates from both the "bilayer', and "monolayer" regions in the isotherm and found the ratio of their measured total thicknesses to be $\sim 1.7$. In the present study, $\mathrm{x}$-ray specular reflectivity $(\mathrm{XR})$ was employed to directly measure the average electron density profiles across the water-PBLG LM-gas interface for both of the regions. The results show that the numbers of molecules per unit area in the two layers of a bilayer are not the same. (ii) The in-plane arrangement of these PBLG rods parallel to the water surface was probed by the GID technique. Evidence for a partial alignment of these rods within the LM plane is provided by observation of a relatively broad peak at a scattered wave vector $(\sim 2 \pi / d)$ that is consistent with the expected $d$-spacing between neighboring parallel $\alpha$-helices. In this connection, the dependence of the interhelix distance on $\Pi$ and $A$, the limited extent of in-plane positional correlations, as well as the incommensurability of the two layers in the bilayer are discussed. (iii) Microscopic lateral inhomogeneities within both PBLG monolayers and bilayers were studied using an x-ray off-specular diffuse scattering (XOSDS) technique. The XOSDS intensities observed from the bilayer have been found to exceed the values theoretically expected for a homogeneous bilayer with thermal capillary wave fluctuations. The excess scattering is explained quantitatively in terms of inhomogeneities in the newly formed second layer.

Finally, one of the issues that motivated the present study is concerned with the effect of sample dispersity on PBLG LM structures. The lyotropic liquid crystalline behavior of conventional polydisperse PBLG in $3 \mathrm{D}$ has been known since the late $1950 \mathrm{~s} ;{ }^{28,52-59}$ in particular, in addition to the usual cholesteric phase, ${ }^{28,52-56,58,59}$ the observations of nematic ${ }^{53,54,60}$ and columnar ${ }^{57}$ phases have been reported. Recently, Tirrell et al. ${ }^{61}$ demonstrated that unlike the polydisperse case, the monodisperse PBLG in both bulk solutions and solution cast films exhibits a smecticlike liquid crystalline order. Their x-ray diffraction results clearly indicated that the smectic layers had a thickness close to the length of these rodlike molecules, and this has been attributed to the narrow size distribution in the monodisperse sample. ${ }^{61}$ The present study extends the investigation of such dispersity effects into 2D. In contrast to the 3D case, both XR and GID results on LMs show very little dependence on the sample dispersity; in particular, no evidence for smectic layering has been found for monodisperse PBLG LMs.

The organization of this paper is as follows: Sec. II describes the experimental methods used. The XOSDS subsection provides a more detailed discussion elucidating a recently developed analysis procedure used to quantitatively distinguish homogeneous and inhomogeneous liquid surfaces. In Sec. III, the experimental results of the $\Pi-A$ isotherm, BAM, and x-ray scattering measurements are presented and discussed. The $\mathrm{X}$-ray subsection is divided into three parts: (i) XR, (ii) GID, and (iii) XOSDS. In Sec. IV, the main conclusions from this study are summarized. 


\section{EXPERIMENTAL DETAILS AND ANALYSIS METHODS}

\section{A. Sample, Langmuir trough, and $\Pi-A$ isotherms}

The polydisperse sample of PBLG [MW 26000 (vis); DP 119, PDI 1.5] was purchased from Sigma Chemical Co. The monodisperse sample [MW 16 700; DP 76] was synthesized using the recombinant DNA method described in $\mathrm{Yu}$ et al. ${ }^{61}$ The PBLG molecule, due to its $\alpha$-helix conformation, resembles a rigid rodlike structure, whose rod diameter is $\sim 13 \AA .^{61}$ The length of the PBLG rod is about $115 \AA$ for the monodisperse sample ${ }^{61}$ and on the order of $150 \AA$ for the polydisperse case.

The Langmuir troughs and the film deposition and compression methods used have been described previously. ${ }^{27,62,63}$ Both the trough and the moveable barrier are made of teflon, and the surface pressure $\Pi$ is measured with a Wilhelmy balance. For all of the measurements, pure water subphase (Milli-Q quality) was used, and the temperature was maintained at $22^{\circ} \mathrm{C}$ to $23^{\circ} \mathrm{C}$. For the $\Pi-A$ isotherm measurements, the sealed aluminum box enclosing the trough assembly was filled with high purity $\mathrm{N}_{2}$ gas. During the x-ray measurements, high purity $\mathrm{He}$ gas was used instead of $\mathrm{N}_{2}$ to reduce background scattering. A PBLG monolayer was prepared by spreading a pure chloroform or 3\%- trifluoroacetic acid/97\%-chloroform mixture (\% by volume) solution of PBLG on water at a specific area equal to or larger than 23 $\AA^{2} /$ monomer. ${ }^{64}$ The PBLG concentration in the spreading solutions used ranged from 0.1 to $0.5 \mathrm{mg} / \mathrm{mL}$, and the volume of the solution spread ranged from 50 to $200 \mu \mathrm{L}$. No significant dependence on the concentration and the spread volume was found in any of the measurements performed during this study.

$\Pi-A$ isotherms were measured using both continuous and relaxation methods. ${ }^{27,62,63}$ The two methods differ on whether or not the film is allowed to relax after each compression step. During a relaxation scan, the surface pressure was monitored every minute while the film relaxed at a given fixed area/monomer $A$. When the surface pressure change over five minutes was less than $0.05 \mathrm{dyn} / \mathrm{cm}$, a final surface pressure measurement was recorded, and the next compression step was taken. In a continuous scan, the film was compressed at a constant rate, and the surface pressure was measured immediately after each compression step. The barrier speeds used in both compression methods corresponded to compression rates ranging from 0.005 to 0.01 $\AA^{2} /$ monomer-s. During x-ray experiments, the continuous method was used for compression from one area of interest to the next, but the film was allowed to relax after the target area/monomer was reached.

\section{B. Brewster-angle microscope (BAM)}

The BAM setup used has been described previously. ${ }^{27,63}$ A $p$-polarized laser beam (argon-ion laser, $\lambda=488 \mathrm{~nm}$ ) strikes the LM surface at the Brewster condition for the bare water surface (an incident angle of 53.3 $3^{\circ}$ from the surface normal). The nonzero reflected intensity caused by the presence of a monolayer was used to image the LM coated surface. The reflected beam was passed through an achromatic

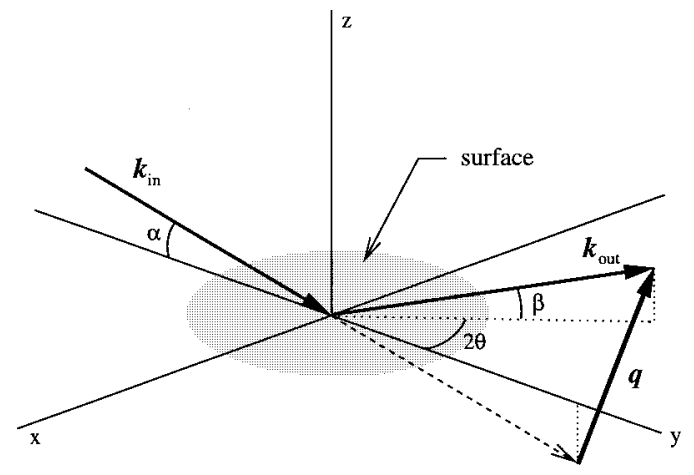

FIG. 1. General x-ray scattering geometry. The surface lies in the $x$-y plane.

lens with a focal length of $175 \mathrm{~mm}$, and the image was recorded by a CCD camera, placed at $\sim 1450 \mathrm{~mm}$ from the lens. With this setup, a surface area of $\sim 0.86 \mathrm{~mm} \times 1.1 \mathrm{~mm}$ on the illuminated LM surface was captured in each image at a magnification of 7.3 and a resolution of $20 \mu \mathrm{m}$.

\section{X-ray scattering techniques}

The $\mathrm{x}$-ray scattering experiments were carried out using the Harvard/BNL liquid surface spectrometer ${ }^{62}$ at Beamline X22B, National Synchrotron Light Source, Brookhaven National Laboratory. The general scattering geometry is illustrated in Fig. 1. Highly collimated monochromatic x-rays of wavelength $\lambda=1.55 \AA$ and incident wave vector $\mathbf{k}_{\text {in }}$ strike the LM surface at an incident angle $\alpha$. The scattered x-rays are characterized by an output wave vector $\mathbf{k}_{\text {out }}$, an angle $\beta$ to the surface and an angle $2 \theta$ to the plane of incidence. The difference between the incident and output wave vectors defines the wave vector transfer, $\mathbf{q}=\mathbf{k}_{\text {out }}-\mathbf{k}_{\text {in }}$. The Cartesian components of $\mathbf{q}$ are

$$
\begin{aligned}
& q_{z}=k[\sin (\alpha)+\sin (\beta)] \\
& q_{x}=k \cos (\beta) \sin (2 \theta) \\
& q_{y}=k[\cos (\beta) \cos (2 \theta)-\cos (\alpha)]
\end{aligned}
$$

where $k=2 \pi / \lambda$. In particular, the component parallel to the LM surface is equal to

$$
\begin{aligned}
q_{x y} & =\sqrt{q_{x}^{2}+q_{y}^{2}} \\
& =k \sqrt{\cos ^{2}(\alpha)+\cos ^{2}(\beta)-2 \cos (\alpha) \cos (\beta) \cos (2 \theta)} .
\end{aligned}
$$

The following scattering techniques were used.

\section{X-ray specular reflectivity (XR)}

In XR, the specularly reflected intensity $I$ is measured as a function of the incident angle $\alpha$ or wave vector transfer $q_{z}=(4 \pi / \lambda) \sin (\alpha)$ along the surface normal while maintaining the specular reflection condition given by $\beta=\alpha$ and $2 \theta$ $=0$ or equivalently $q_{x y}=0$. A pair of crossed Huber slits of width $W=3 \mathrm{~mm}$ and height $H=2.5 \mathrm{~mm}$, located approximately $L \sim 620 \mathrm{~mm}$ from the sample center, were placed in front of a NaI scintillation detector to define its angular acceptance $\delta \beta \sim H / L \sim 0.23^{\circ}$ and $\delta(2 \theta) \sim W / L \sim 0.28^{\circ}$. The background due to bulk scattering from the subphase and 
scattering from sample cell windows and gas in the beam path was accounted for by subtracting the intensities measured at $\beta=\alpha$ with $2 \theta$ offsets of $\pm 0.3^{\circ}$. The result of the subtraction, i.e., $\Delta I(\alpha)=I(\alpha, 2 \theta=0)-\left(\frac{1}{2}\right)\left[I\left(\alpha,+0.3^{\circ}\right)\right.$ $\left.+I\left(\alpha,-0.3^{\circ}\right)\right]$, was normalized to the incident beam intensity $I_{0}$ to obtain the measured specular reflectivity $R\left(q_{z}\right)$.

For water as the subphase, the critical wave vector for total reflection is equal to $q_{c}=0.0218 \AA^{-1}$, and the corresponding critical angle of incidence is $\alpha_{c}=0.154^{\circ}$ for $\lambda$ $=1.55 \AA$. For $q_{z} / q_{c}>4 \sim 5\left(q_{z}>\sim 0.1 \AA^{-1}\right.$ for water $)$, the specular reflectivity $R\left(q_{z}\right)$ from a macroscopically homogeneous surface is well described by the Born approximation expression $^{4,5,65}$

$$
\frac{R\left(q_{z}\right)}{R_{F}\left(q_{z}\right)} \cong\left|\int_{-\infty}^{+\infty} d z \frac{d}{d z}\left[\frac{\langle\rho(z)\rangle}{\rho_{\infty}}\right] e^{-i q_{z} z}\right|^{2},
$$

where $\langle\rho(z)\rangle$ is the average electron density profile ("average", over the appropriate coherence lengths in the $x$-y plane) across the interface at height $z$ along the surface normal, and $\rho_{\infty}$ is the electron density in the bulk subphase $\left(\rho_{\infty}\right.$ $=0.334$ electrons $/ \AA^{3}$ for water $) . R_{F}\left(q_{z}\right)$ is the Fresnel reflectivity expected from an ideally flat and sharp bulk-gas interface, which can be expressed as

$$
R_{F}\left(q_{z}\right) \cong\left|\frac{q_{z}-\sqrt{q_{z}^{2}-q_{c}^{2}}}{q_{z}+\sqrt{q_{z}^{2}-q_{c}^{2}}}\right|^{2} .
$$

For $q_{z} \leqslant q_{c}$, Eq. (4) results in total external reflection $\left(R_{F}\left(q_{z}\right)=1\right)$, and for $q_{z} \gg q_{c}$, it decays algebraically as $R_{F}\left(q_{z}\right) \approx\left(q_{c} / 2 q_{z}\right)^{4}$.

\section{Grazing incidence diffraction (GID)}

For GID, x-rays are incident on the surface at an angle $\alpha<\alpha_{c}$, such that the total reflection condition is maintained. This results in an evanescent wave on the bulk side of the interface and thereby suppresses the scattering from the bulk relative to the surface. The scattered intensity is measured as a function of $2 \theta$ ( or $q_{x y}$ ) while maintaining $\beta \sim 0$ (or $q_{z} \sim 0$ ). Structures periodic in the surface plane with a repeat distance $d$ result in a diffraction peak at $q_{x y}=2 \pi / d$. Most of the GID data were collected using a fixed incident angle of $\alpha$ $=0.12^{\circ}$ and Soller slits in front of the NaI scintillator detector. The Soller slits had a horizontal angular acceptance of $\delta(2 \theta) \sim 0.16^{\circ}$, corresponding to an in-plane FWHM (full width at half maximum) resolution of $\delta q_{x y} \sim 0.012 \AA^{-1}$. The vertical opening of the Soller slits (limited to $18 \mathrm{~mm}$ ) corresponded to the integration of scattered signals over $\Delta \beta$ $\sim 1.7^{\circ}$ or $\Delta q_{z} \sim 0.12 \AA^{-1}$.

As a result of the finite width of the horizontal detector opening $(W=6 \mathrm{~mm})$ and the narrow $2 \theta$ resolution of the Soller slits, when $2 \theta$ is large enough, the detector only views a fraction of the illuminated path $\left(L_{f} \sim 40 \mathrm{~mm}\right)$ along the LM surface. For $2 \theta>W / L_{f} \sim 8.6^{\circ}$ (or $q_{x y}>\sim 0.6 \AA^{-1}$ ), this fraction varies as $1 / \sin (2 \theta)$, yielding a measured intensity proportional to $q_{x y}^{-1} \times(2 \mathrm{D}$ structure factor). In addition to the specularly reflected signal, there is also a background due to scattering of the incident and totally reflected beams by the entrance and exit Kapton windows of the sample cell (separated by $L_{w}=16.5 \mathrm{~cm}$ along the beam) as well as from the gas in the beam path. Fortunately, for $2 \theta>W / L_{w}=2.1^{\circ}$ (or $q_{x y}>0.15 \AA^{-1}$ ), the detector resolution screens out the scattering from the two windows and the observed background is almost entirely due to the He gas and water vapor above the surface. The measured intensity from this also varies roughly as $q_{x y}^{-1}$ due to the decrease in the length of the beam path viewed by the detector.

For rod scans, ${ }^{5}$ the $q_{x y}$ (or $2 \theta$ ) position of the detector is held at the center of a GID peak, and the scattered intensities along $q_{z}$ (or $\beta$ ), which depend on molecular scale density correlations normal to the surface, are measured. However, unlike XR, they are sensitive only to the in-plane ordered regions of the surface that give rise to the GID peak. For a LM with no molecular tilt, the intensity falls slowly with $q_{z}$ on the scale of $l^{-1}$, where $l$ is the molecular length along the surface normal. In the rod scan experiment, the data were collected using a Braun position-sensitive linear detector with a quartz wire along the $\beta$-direction. The background, which was nearly all due to scattering from the He gas and water vapor, was eliminated by subtracting similar scans that only differed in that the LM surface was lowered to $2 \mathrm{~mm}$ below the incident beam.

\section{X-ray off-specular diffuse scattering (XOSDS) from liquid surfaces}

In a typical small-angle XOSDS experiment, the intensity scattered from a given surface is measured within the plane of incidence $\left(2 \theta=0\right.$ or $\left.q_{x}=0\right)$ at non-specular conditions, i.e., $\alpha \neq \beta$ or $q_{y} \neq 0$. The nonzero $q_{y}$ component of the wave vector transfer parallel to the surface typically ranges from $10^{-5}$ to $10^{-2} \AA^{-1}$, which is limited by the detector resolution and low counting rates, respectively. Therefore, XOSDS is sensitive to lateral density fluctuations over the surface on submicron length scales, including interfacial height fluctuations.

In this study, a $\beta$-scan method was used in which the scattered intensity $I(\alpha, \beta, 2 \theta=0)$ was measured as a function of $\beta$ at constant $\alpha$. Since $q_{z}$ is varied simultaneously with $q_{y}, \beta$-scans are also sensitive to the density profile along the surface normal. The experimental setup was identical to that for XR except that a vertical detector slit size of $H$ $=1.0 \mathrm{~mm}$ was used. This setup provided angular detector resolutions of $\delta \beta=H / L=0.092^{\circ}$ and $\delta(2 \theta)=W / L=0.28^{\circ}$, which are related to reciprocal space resolutions through $\delta q_{y} \sim(2 \pi / \lambda) \sin (\beta) \delta \beta$ and $\delta q_{x} \sim(2 \pi / \lambda) \delta(2 \theta)$. As in XR, background intensities measured with $2 \theta$ offsets of $\pm 2 \theta_{b}$ $= \pm 0.3^{\circ}$ were subtracted from the intensity measured at $2 \theta$ $=0$ and the same $(\alpha, \beta)$. The result was normalized to the incident beam intensity $I_{0}$ to obtain the measured normalized intensity difference

$$
\begin{aligned}
\frac{\Delta I(\alpha, \beta)}{I_{0}}= & \frac{1}{I_{0}}\left\{I(\alpha, \beta, 2 \theta=0)-\frac{1}{2}\left[I\left(\alpha, \beta, 2 \theta=+2 \theta_{b}\right)\right.\right. \\
& \left.\left.+I\left(\alpha, \beta, 2 \theta=-2 \theta_{b}\right)\right]\right\}
\end{aligned}
$$

A characteristic feature of liquid surfaces, including LM systems, is the presence of capillary waves, that is, thermally excited fluctuations in the liquid-vapor interfacial height 
$h\left(r_{x y}\right)$, which give rise to thermal diffuse scattering (TDS). The capillary waves cause the interfacial height-height correlation function $g\left(r_{x y}\right)=\left\langle\left[h\left(r_{x y}\right)-h(0)\right]^{2}\right\rangle$ to vary logarithmically with the distance $r_{x y}$ along the surface ${ }^{66-68}$ for $r_{x y}$ smaller than a gravitationally imposed cutoff $(\sim \mathrm{mm})$ and large compared to the molecular size $d(\sim \AA)$. Consequently, for $\eta=\left(k_{B} T / 2 \pi \gamma\right) q_{z}^{2}<2$, where $\gamma$ is the surface tension, the scattering cross section is characterized by an algebraic singularity of the form $1 / q_{x y}^{2-\eta}$, without a true specular term $\left[\sim \delta^{(2)}\left(\mathbf{q}_{x y}\right)\right]{ }^{66,67}$ Because of this power-law behavior, the capillary wave TDS is a primary source of small-angle XOSDS from liquid surfaces. Therefore, in order to probe noncapillary lateral inhomogeneities occurring in a LM film, their contribution to the XOSDS intensities needs to be quantitatively separable from the capillary-wave TDS.

A given LM is laterally homogeneous if the heights of all interfaces (water-layer, layer-gas, etc.) are conformal with the capillary fluctuations and if there is no density variation within the surface. In such cases, the local electron density $\rho_{T=0}\left(\mathbf{r}_{x y}, z^{\prime}\right)$, which can be defined conceptually by considering a nonuniform frame in which capillary fluctuations are absent [i.e., $\left.z^{\prime}=z-h\left(r_{x y}\right)\right]$, is equal to its lateral average $\left\langle\rho_{T=0}\left(z^{\prime}\right)\right\rangle$. This average "intrinsic" or local electron density profile $\left\langle\rho_{T=0}\left(z^{\prime}\right)\right\rangle$ is to be distinguished from the total average density profile $\langle\rho(z)\rangle$ defined in Eq. (3), which is obtained from the convolution of $\left\langle\rho_{T=0}\left(z^{\prime}\right)\right\rangle$ with the capillary wave height distribution $\left\{h\left(r_{x y}\right)\right\}$. It has recently been shown that for small-angle scattering from a homogeneous liquid surface, a properly normalized form of the differential cross section is given by ${ }^{69,70}$

$$
\begin{aligned}
\frac{1}{A_{0}}\left(\frac{d \sigma}{d \Omega}\right)_{\mathrm{hmg}} \approx & \frac{1}{16 \pi^{2}}\left(\frac{q_{c}}{2}\right)^{4} \frac{T_{F}(\alpha) T_{F}(\beta)}{q_{z}^{2} \sin (\alpha)} \\
& \times\left|\Phi_{0}\left(q_{z}\right)\right|^{2} \frac{2 \pi \eta}{q_{x y}^{2}}\left(\frac{q_{x y}}{q_{\max }}\right)^{\eta},
\end{aligned}
$$

for $\eta=\left(k_{B} T / 2 \pi \gamma\right) q_{z}^{2}<2$, where $A_{0}$ is the cross-sectional area of the incident beam, and $T_{F}(\alpha)=\left(2 \alpha / \alpha_{c}\right)^{2} \sqrt{R_{F}(\alpha)}$ is the Fresnel transmission factor. ${ }^{66}$ The upper cutoff wave vector $q_{\max }$ is determined by the condition that the number of capillary wave modes is on the order of the number of molecules per unit surface area. This is equivalent to fixing $q_{\max } \sim 2 \pi / d$, where $d$ is on the order of the intermolecular distance. A surface structure factor $\Phi_{0}\left(q_{z}\right)$ is defined as ${ }^{69,70}$

$$
\Phi_{0}\left(q_{z}\right)=\int d z \frac{d}{d z}\left[\frac{\left\langle\rho_{T=0}(z)\right\rangle}{\rho_{\infty}}\right] \exp \left(-i q_{z} z\right) .
$$

Therefore, apart from the effect of layering and local interfacial diffuseness contained in $\Phi_{0}\left(q_{z}\right)$, scattering from a homogeneous LM is still described by the characteristic power law $1 / q_{x y}^{2-\eta}$ of the capillary-wave TDS.

If the LM is laterally inhomogeneous, the local electron density deviates from the lateral average so that $\delta \rho_{T=0}\left(\mathbf{r}_{x y}, z^{\prime}\right) \equiv \rho_{T=0}\left(\mathbf{r}_{x y}, z^{\prime}\right)-\left\langle\rho_{T=0}\left(z^{\prime}\right)\right\rangle \neq 0$, and there will be additional scattering in excess of Eq. (6). This leads to a nonzero second term in the differential cross section, whose general form is given by

$$
\begin{aligned}
\frac{1}{A_{0}}\left(\frac{d \sigma}{d \Omega}\right)_{\text {inhmg }}= & \frac{1}{16 \pi^{2}}\left(\frac{q_{c}}{2}\right)^{4} \frac{1}{A_{0}} \mid \int d^{3} \mathbf{r} \exp (-i \mathbf{q} \cdot \mathbf{r}) \\
& \times\left.\exp \left(-i q_{z} h\left(r_{x y}\right)\right) \frac{\delta \rho_{T=0}(\mathbf{r})}{\rho_{\infty}}\right|^{2} .
\end{aligned}
$$

In general, the normalized intensity $I / I_{0}$ is equal to the convolution of the differential cross section with an appropriate instrumental resolution function $\Xi$

$$
\begin{aligned}
\frac{I(\alpha, \beta, 2 \theta)}{I_{0}}= & \int d \beta^{\prime} \int d\left(2 \theta^{\prime}\right) \Xi_{\Omega}\left(\beta-\beta^{\prime} ; 2 \theta-2 \theta^{\prime}\right) \\
& \times \frac{1}{A_{0}} \frac{d \sigma}{d \Omega}\left(\alpha, \beta^{\prime}, 2 \theta^{\prime}\right),
\end{aligned}
$$

or equivalently, using the approximation $d \Omega \approx d \beta d(2 \theta)$ $\approx d^{2} \mathbf{q}_{x y} /\left[k^{2} \sin (\beta)\right]$

$$
\frac{I(\mathbf{q})}{I_{0}}=\int \frac{d^{2} \mathbf{q}_{x y}^{\prime}}{k^{2} \sin (\beta)} \Xi_{q}\left(\mathbf{q}_{x y}-\mathbf{q}_{x y}^{\prime}\right) \frac{1}{A_{0}} \frac{d \sigma}{d \Omega}\left(\mathbf{q}_{x y}^{\prime}\right) .
$$

For $\alpha \gg \alpha_{c}$, the physical size of the detector opening is much larger than the projection of the illuminated sample area onto the plane of the detector. Therefore, the simple and reasonable choice for $\Xi$ is a rectangular resolution function whose center and area correspond to the nominal detector position and the angular acceptance defined by the detector slits, respectively

$$
\begin{aligned}
\Xi_{\Omega}(\Delta \beta ; \Delta(2 \theta)) \\
\quad= \begin{cases}1 & \text { if }|\Delta \beta| \leqslant \delta \beta / 2,|\Delta(2 \theta)| \leqslant \delta(2 \theta) / 2 \\
0 & \text { otherwise }\end{cases}
\end{aligned}
$$

or equivalently

$$
\Xi_{q}\left(\Delta q_{x}, \Delta q_{y}\right)=\left\{\begin{array}{ll}
1 & \text { if }\left|\Delta q_{x}\right| \leqslant \delta q_{x} / 2,\left|\Delta q_{y}\right| \leqslant \delta q_{y} / 2 \\
0 & \text { otherwise }
\end{array} .\right.
$$

By substituting Eq. (6) into Eq. (9), carrying out the convolution with the slit-defined resolution function from Eq. (10) numerically, and taking the same intensity difference as in Eq. (5), one can calculate the homogeneous capillary-wave contribution $\left[\Delta I(\alpha, \beta) / I_{0}\right]_{\mathrm{hmg}}$ to the normalized XOSDS intensity difference $\Delta I(\alpha, \beta) / I_{0} \cdot{ }^{69-71}$ The physical parameters $(T, \gamma)$ and detector resolutions are known, and $q_{\max }$ can be estimated from the size of the LM forming molecules. Moreover, since Eq. (6) is also valid for $\mathrm{XR}$, the factor $\Phi_{0}\left(q_{z}\right)$, or the intrinsic profile $\left\langle\rho_{T=0}(z)\right\rangle$, can be determined by fitting $\left[\Delta I(\alpha, \beta=\alpha) / I_{0}\right]_{\mathrm{hmg}}$ to the measured specular reflectivity $R\left(q_{z}\right) \cdot{ }^{69,70}$ Consequently, the homogeneous contribution $\left[\Delta I(\alpha, \beta) / I_{0}\right]_{\mathrm{hmg}}$ can be calculated with no adjustable parameters. It follows that comparison between the theoretical curve $\left[\Delta I(\alpha, \beta) / I_{0}\right]_{\mathrm{hmg}}$ and the measured data $\Delta I(\alpha, \beta) / I_{0}$ provides an unambiguous test of the homogeneity for a given LM. Any excess scattering $\Delta I / I_{0}-\left[\Delta I / I_{0}\right]_{\mathrm{hmg}}>0$ is a measure of surface inhomogeneities $\delta \rho_{T=0}\left(r_{x y}, z\right) \neq 0$, and can be analyzed in terms of the convolution of Eq. (8) with the same resolution functions. 

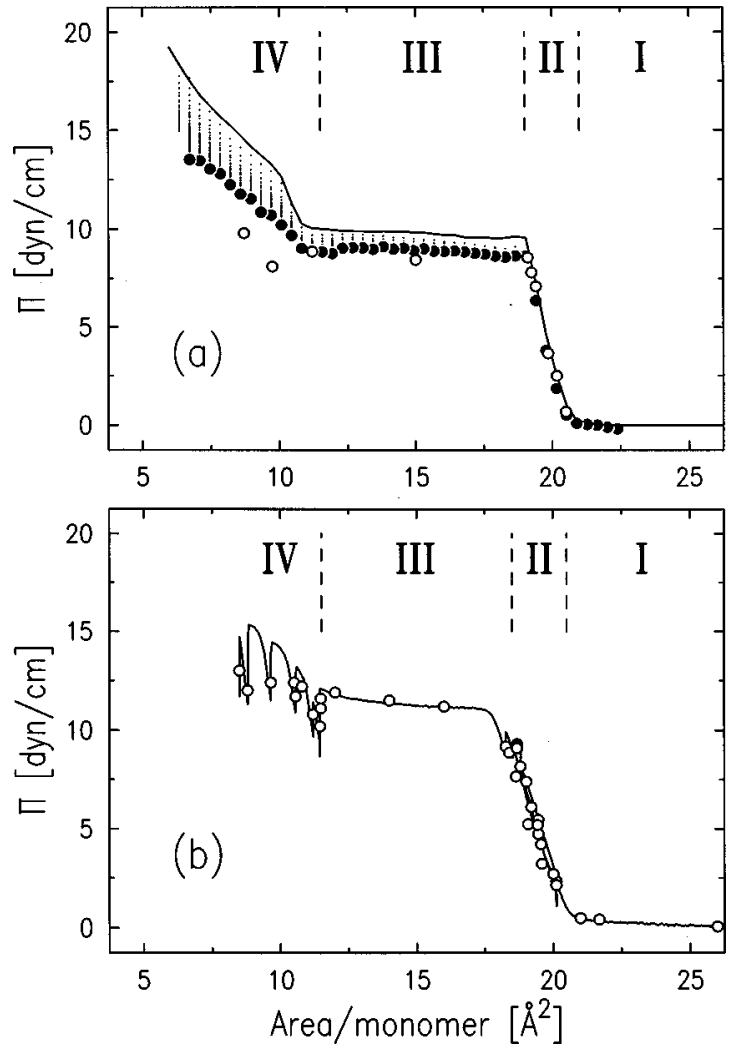

FIG. 2. $\Pi-A$ isotherms at $T=23{ }^{\circ} \mathrm{C}$ for (a) polydisperse and (b) monodisperse PBLG films. In (a), the solid line $(-)$ is a continuous isotherm, and the filled circles $(\bullet)$ correspond to a relaxation isotherm. The dots $(\cdots)$ show intermediate surface pressures measured during relaxation at fixed $A$. In (b), the solid line (-) is a set of continuous compression/expansion scans taken on Film-1 during the x-ray scattering experiment. In both (a) and (b), the open circles $(O)$ indicate the points at which $x$-ray scattering measurements were carried out.

\section{4. $X$-ray radiation damage}

During the x-ray experiments on PBLG LMs, changes in the scattered intensity were observed when the maximum incident flux (on the order of $10^{9}$ photons/s) was used. For GID, in which $\alpha$ is small and, therefore, the incident beam is spread over a larger surface area, radiation-induced changes (often evidenced by the disappearance of a peak) occurred only after exposing the same spot of the film for many hours at full intensity. For XR and XOSDS, at the largest values of $\alpha$ measured, the use of high flux led to changes in the reflected intensities after less than $30 \mathrm{~min}$. Subsequently, special care was taken to minimize radiation damage by limiting the x-ray exposure through absorbers and carefully monitoring the scattered signals. There was no indication of film damage in any of the results presented here.

\section{RESULTS AND DISCUSSION}

\section{A. $\Pi-A$ isotherms and surface imaging by BAM}

Representative $\Pi-A$ isotherms are shown in Fig. 2(a) for polydisperse and in Fig. 2(b) for monodisperse PBLG. BAM images obtained from a polydisperse film are summarized in Figs. 3 and 4. In Fig. 2(a), the solid line and the filled circles correspond to a continuous and a relaxation isotherm, respectively. The solid line in Fig. 2(b) is a continuous scan taken during the $\mathrm{x}$-ray scattering experiments. In both figures, the $\Pi-A$ positions at which $\mathrm{x}$-ray measurements were carried out are indicated with open circles. The shown isotherms are very similar to those obtained previously by others for polydisperse samples. ${ }^{12,13,18,25,46}$ As indicated in Fig. 2, the П- $A$ phase diagram can be divided into four main regions, each exhibiting a different surface pressure variation with compression.

In Region I ( $A>\sim 21 \AA^{2} /$ monomer), the surface pressure remains zero as the film is compressed. This behavior is due to an incomplete surface coverage, which is clearly evident in BAM images from Region I [Figs. 3(a)-3(c)]. As deposited, LM-coated regions coexist with regions of bare water surface. A typical image Fig. 3(a) reveals that a LMcoated region itself consists of a network of PBLG islands (bright) with small gaps of bare water surface in between (dark). In an epifluorescence microscopy study, Lavigne et al. also observed an aggregation of PBLG molecules into 2D islands at low surface density. ${ }^{25}$ As a result, compression in Region I only leads to a reduction in the area of the gaps between PBLG islands, as illustrated in a series of images Figs. 3(a)-3(c) taken at $A=31,25,23 \AA^{2} /$ monomer. This type of compression is consistent with the absence of measurable surface pressure since it does not alter the microscopic structure within the islands themselves. Another important BAM observation is that the monolayer is solidlike as indicated by the intricate shapes of the island-water boundaries which would not be expected for a fluid monolayer.

Region II $\quad\left(\sim 18.5 \AA^{2} /\right.$ monomer $<A<\sim 21 \AA^{2} /$ monomer) is characterized by an increase in the surface pressure above $\Pi \sim 0$. Given the PBLG rod diameter of about $13 \AA^{61}$ and the $\alpha$-helix pitch of $1.5 \AA$ /monomer along the rod axis, one expects a limiting area/monomer of $A_{\text {lim }} \sim 19.5$

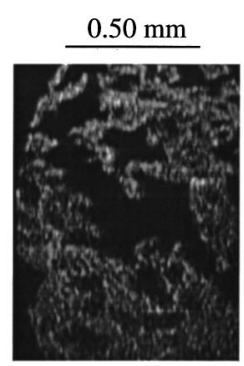

(a)

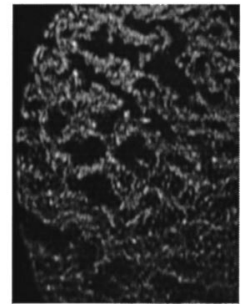

(b)

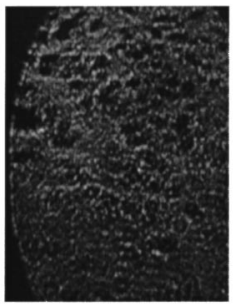

(c)

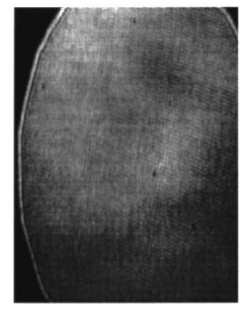

(d)
FIG. 3. BAM images taken at $A=$ (a) 31, (b) 25, (c) 23, and (d) $20 \AA^{2} /$ monomer. The images (a)-(c) are in Region I of the П- $A$ isotherm, while (d) is in Region II. 


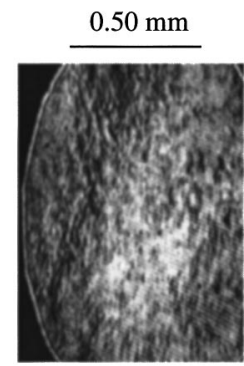

(a)

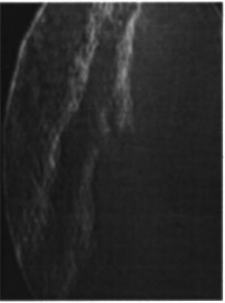

(b)

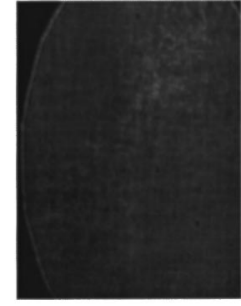

(c)

FIG. 4. BAM images taken at $A=13.3 \AA^{2} /$ monomer in Region III. The moveable barrier and the trough edge on the opposite side, both of which run parallel to the vertical edges of the images, are located $\sim 6.5 \mathrm{~cm}$ to the left and $5 \mathrm{~cm}$ to the right of image (b), respectively. Images (a) and (c) were taken from spots that were less than $1 \mathrm{~cm}$ away from the image (b) spot, with (a) being on the barrier side and (c) being on the opposite side. The sensitivity of the CCD camera used here [the same for (a)-(c)] is lower than that in Fig. 3.

$\AA^{2} /$ monomer for a close packed monolayer of PBLG rods lying down parallel to the water-gas interface. ${ }^{25}$ The fact that this $A_{\text {lim }}$ value falls into Region II, where the $\Pi$ increase is observed, provides indirect evidence for both the single molecule thickness of the film in Regions I and II and the parallel-to-surface orientation of the PBLG rods. In Region II, the entire surface is uniformly covered with the PBLG LM. A typical BAM image Fig. 3(d), taken at $A$ $=20 \AA^{2} /$ monomer, also shows that the LM surface has become more or less homogeneous. Since the bare areas observed in Region I are now absent, compression in Region II should result in a microscopic reduction in the intermolecular distance within the 2D plane.

Region III $\left(\sim 11.5 \AA^{2} /\right.$ monomer $<A<\sim 18.5 \AA^{2} /$ monomer) of the isotherm is characterized by a plateau of infinite compressibility $\left(\chi=-A^{-1} \cdot d A / d \Pi=\infty\right)$, which signifies a first-order phase transition between two coexisting phases. Since the ratio of the area/monomer values at the two ends of this coexistence region is close to two, the second phase coexisting with the monolayer phase has been assumed to be a bilayer. ${ }^{11,12,18,25}$ Further compression into Region IV ( $A$ $<\sim 11.5 \AA^{2} /$ monomer) results in a surface pressure increase in the continuous isotherms. However, the relaxation effect is now more significant. When the film is left to relax at a constant area/monomer in this region, surface pressure decreases almost to the plateau value. As indicated by the open circles in Fig. 2, x-ray measurements in Region IV were made on relaxed films.

Figure 4 shows BAM images taken at $A=13.3 \AA^{2} /$ monomer in Region III. As shown in Fig. 4(b), a region of inhomogeneity (confined to less than $1 \mathrm{~cm}$ along the compression direction) was found in which ridge or steplike morphologies ran more or less parallel to the moveable barrier (i.e., perpendicular to the compression direction). The images [see Fig. 4(c)] taken from the stationary edge side of this inhomogeneous region are very similar to those obtained for the monolayer in Region II. However, the intensity reflected from the moveable barrier side of the surface [see Fig. 4(a)] is consistently higher than that from the other side. These observations indicate that the monolayer coexists with
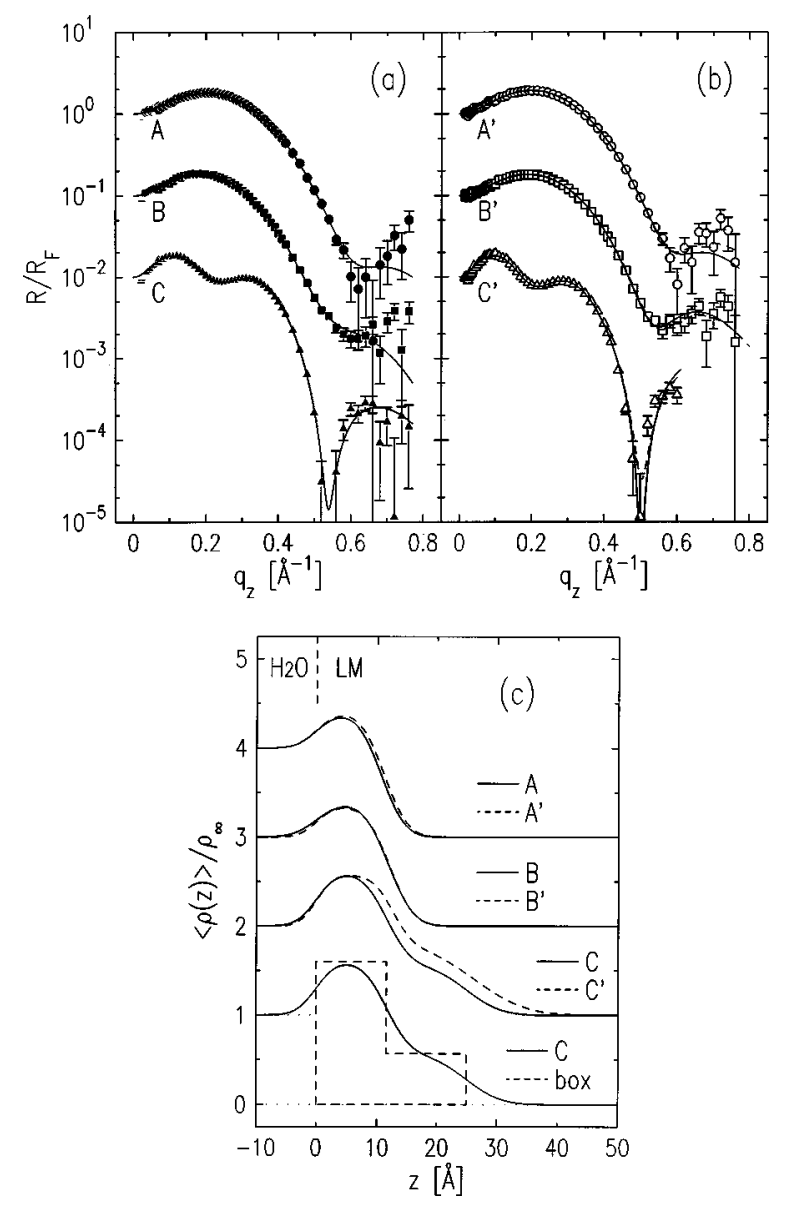

FIG. 5. Measured specular reflectivity normalized to the Fresnel reflectivity of an ideally flat and sharp water-gas interface for (a) monodisperse and (b) polydisperse PBLG films. In (a), the shown $R / R_{F}$ data were obtained at $A$ (O): $21.7 \AA^{2} /$ monomer, $0.4 \mathrm{dyn} / \mathrm{cm} ; B(\mathbf{\square}): 18.7 \AA^{2} /$ monomer, $9.3 \mathrm{dyn} / \mathrm{cm}$; $C(\boldsymbol{\Delta}): 11.5 \AA^{2} /$ monomer, $11.6 \mathrm{dyn} / \mathrm{cm}$. In (b), the data were obtained at $A^{\prime}$ (○): $20.2 \AA^{2} /$ monomer, $2.5 \mathrm{dyn} / \mathrm{cm} ; B^{\prime}(\square): 19.2 \AA^{2} /$ monomer, $7.8 \mathrm{dyn} / \mathrm{cm}$; $C^{\prime}(\triangle): 9.7 \AA^{2} /$ monomer, $8.8 \mathrm{dyn} / \mathrm{cm}$. The solid curves $(-)$ in (a) and (b) are best fits given by Eq. (3) and the box-model total average electron density profiles $\langle\rho(z)\rangle / \rho_{\infty}$ shown in (c). The dashed lines (---) in (b) for the polydisperse film are best fits based on Eq. (6) and the box-model average local electron density profiles $\left\langle\rho_{T=0}(z)\right\rangle / \rho_{\infty}$ shown in the insets in Figs. 12(a) and 13(a).

a thicker phase in Region III. Moreover, the coexistence is not uniform over the surface. While the stationary edge side of the surface is mostly still a monolayer, the film on the moveable barrier side seems to be dominated by a thicker phase. This suggests that the formation of the second thicker phase starts preferably at the moving barrier and grows parallel to the compression direction. ${ }^{22}$ Evidence that this second phase is in fact a bilayer is provided by the XR results to be discussed below.

During the BAM study, birefringence effects were also investigated by placing an analyzing polarizer after the imaging lens. However, no clear evidence was found for optical anisotropy within the PBLG films. This seems to suggest a lack of long-range orientational order and the absence of large oriented domains within the film. However, it may also be due to a relatively small difference between the refractive indices in the directions parallel and perpendicular to the molecular axes. ${ }^{38,39,41,72}$ 
TABLE I. The list of best-fit parameters used to fit the measured $R / R_{F}$ data, where the fits are based on Gaussian-smeared box models for the average electron density profile $\langle\rho(z)\rangle / \rho_{\infty}$ across the water-PBLG-gas interface. The parameter designations are as follows. (a) Single-layer box model for PBLG monolayers: Layer thickness, $l_{1}$; relative electron density in the layer, $\phi_{1}=\rho_{1} / \rho_{\infty}$; water-layer and layer-gas interfacial roughness, $\sigma_{w 1}, \sigma_{1 g}$. (b) Double-layer box model for PBLG bilayers: Layer-1 and layer-2 thickness, $l_{1}, l_{2}$; layer-1 and layer-2 relative electron density, $\phi_{1}=\rho_{1} / \rho_{\infty}, \phi_{2}=\rho_{2} / \rho_{\infty}$; water-layer-1, layer-1-layer-2, and layer-2gas interfacial roughness, $\sigma_{w 1}, \sigma_{12}, \sigma_{2 g}$.

\begin{tabular}{cccccccc}
\hline \hline \multicolumn{7}{c}{ (a) Single-layer box models for monolayers } \\
& Dispersity & $\begin{array}{c}A \\
{\left[\AA^{2} / \text { monomer }\right]}\end{array}$ & $\begin{array}{c}\langle\Pi\rangle \\
{[\mathrm{dyn} / \mathrm{cm}]}\end{array}$ & $\phi_{1}=\rho_{1} / \rho_{\infty}$ & $\begin{array}{c}l_{1} \\
{[\AA]}\end{array}$ & $\begin{array}{c}\sigma_{w 1} \\
{[\AA]}\end{array}$ & $\begin{array}{c}\sigma_{1 g} \\
{[\AA]}\end{array}$ \\
\hline$A$ & mono & 21.7 & 0.4 & $1.39 \pm 0.09$ & $10.6 \pm 1.0$ & $2.67 \pm 0.5$ & $3.14 \pm 0.5$ \\
$B$ & mono & 18.7 & 9.3 & $1.39 \pm 0.06$ & $11.8 \pm 0.8$ & $3.12 \pm 0.4$ & $3.35 \pm 0.23$ \\
$A^{\prime}$ & poly & 20.2 & 2.5 & $1.40 \pm 0.08$ & $11.1 \pm 0.9$ & $2.66 \pm 0.5$ & $3.14 \pm 0.5$ \\
$B^{\prime}$ & poly & 19.2 & 7.8 & $1.34 \pm 0.04$ & $12.1 \pm 0.5$ & $2.31 \pm 0.4$ & $3.18 \pm 0.2$
\end{tabular}

(b) Double-layer box models for bilayers

\begin{tabular}{|c|c|c|c|c|c|c|c|c|c|c|}
\hline & Disp. & $\begin{array}{c}A \\
{\left[\AA^{2} / \text { mon. } .\right]}\end{array}$ & $\begin{array}{c}\langle\Pi\rangle \\
{[\mathrm{dyn} / \mathrm{cm}]}\end{array}$ & $\phi_{1}$ & $\phi_{2}$ & $\begin{array}{c}l_{1} \\
{[\AA ̊]}\end{array}$ & $\begin{array}{c}l_{2} \\
[\AA]]\end{array}$ & $\begin{array}{l}\sigma_{w 1} \\
{[\AA]}\end{array}$ & $\begin{array}{l}\sigma_{12} \\
{[\AA]}\end{array}$ & $\begin{array}{l}\sigma_{2 g} \\
{[\AA]}\end{array}$ \\
\hline \multirow[t]{2}{*}{$C$} & mono & 11.5 & 11.6 & 1.60 & 0.57 & 11.6 & 13.4 & 2.61 & 3.17 & 4.52 \\
\hline & & & & \pm 0.10 & \pm 0.10 & \pm 0.6 & \pm 0.8 & \pm 0.7 & \pm 0.9 & \pm 1.0 \\
\hline \multirow[t]{2}{*}{$C^{\prime}$} & poly & 9.7 & 8.8 & 1.57 & 0.81 & 12.5 & 13.6 & 2.24 & 2.64 & 6.76 \\
\hline & & & & \pm 0.04 & \pm 0.13 & \pm 0.6 & \pm 0.6 & \pm 0.5 & \pm 0.6 & \pm 0.9 \\
\hline
\end{tabular}

\section{B. X-ray scattering results}

\section{1. $X$-ray specular reflectivity $(X R)$}

Representative reflectivity data are shown in Fig. 5(a) for monodisperse and Fig. 5(b) for polydisperse PBLG Langmuir films. The three $R / R_{F}$ curves in each figure correspond to: A low- $\Pi$ film in Region II, near the I/II boundary $\left(A, A^{\prime}\right)$; a high- $\Pi$ film in Region II, near the II/III boundary $\left(B, B^{\prime}\right)$; and a film in Region IV, on the high-density side of the coexistence region $\left(C, C^{\prime}\right)$. The results are nearly independent of the sample dispersity. The oscillation rate (in $q_{z}$ ) of the $R / R_{F}$ curves $C$ and $C^{\prime}$ is higher by about a factor of 2 than that for the curves $A, B, A^{\prime}$, and $B^{\prime}$. This implies that PBLG films in Region IV are about twice as thick as those in Region II.

Quantitative fitting of each reflectivity curve was done by constructing a simple "box" model ${ }^{4,5,27}$ for the relative average electron density profile $\langle\rho(z)\rangle / \rho_{\infty}$ across the waterPBLG-gas interface. In a single-layer box model, a layer of thickness $l_{1}$ and density $\rho_{1} / \rho_{\infty}=\phi_{1}$ is assumed between the bulk subphase below $\left(\rho / \rho_{\infty}=1\right)$ and the gas above $\left(\rho / \rho_{\infty}\right.$ $=0)$, and the water-layer and layer-gas interfaces are smeared out with Gaussian roughnesses $\sigma_{w 1}$ and $\sigma_{1 g}$, respectively. In a double-layer box model, a second layer of thickness $l_{2}$ and density $\rho_{2} / \rho_{\infty}=\phi_{2}$ is added on top of the first layer; the Gaussian roughnesses for the layer-1-layer-2 and layer-2-gas interfaces are designated as $\sigma_{12}$ and $\sigma_{2 g}$, respectively.

Using these models, the $R / R_{F}$ data have been fit to the Born approximation expression [Eq. (3)] for $q_{z} \geqslant 0.1 \AA^{-1}$. The single-layer model was used for the PBLG films in Region II $\left(A, B ; A^{\prime}, B^{\prime}\right)$, while the double-layer model was necessary to obtain good fits in Region IV $\left(C, C^{\prime}\right)$. The best-fit $R / R_{F}$ curves are shown as the solid lines in Figs. 5(a) and $5(\mathrm{~b})$. The corresponding density profiles $\langle\rho(z)\rangle / \rho_{\infty}$ are shown in Fig. 5(c), and the best-fit parameters are listed in Table I. As evident in Figs. 5(a) and 5(b), the reflectivity data are well described by the simple box models. In some cases, there are small residual deviations between the data and the fits at large $q_{z}$ (where the error bars are large). Although the use of more sophisticated models or increased number of parameters could further improve the fitting, the improvement would only appear in fine structural details that would not affect the principal physical interpretations.

It is evident from Fig. 5(c) and Table I(a) that both the monodisperse and polydisperse PBLG films in Region II $\left(A, B ; A^{\prime}, B^{\prime}\right)$ are monolayers with their layer thicknesses $l_{1}$ being comparable to the PBLG rod diameter of $\sim 13 \AA$, as expected. Theoretically, since the PBLG molecule contains 116 electrons/monomer, one expects the surface density of electrons originating from a PBLG monolayer to increase from 5.4 to 6.3 electrons $/ \AA^{2}$ as the specific area is reduced from $A=21.5$ to $18.5 \AA^{2} /$ monomer. Experimentally, the surface electron density is given roughly by the integrated area under the single-layer box (i.e., $\sim \rho_{\infty} \cdot \phi_{1} \cdot l_{1}$ ). The latter experimentally derived estimates of surface electron densities are within $10 \%$ of the theoretically expected values, indicating that the values for the monolayer thickness and density parameters are physically reasonable. As for the roughness parameters, for all of the PBLG monolayers in Region II, the layer-gas interface $\left(\sigma_{1 g}\right)$ is just slightly rougher or more diffuse than the water-layer interface $\left(\sigma_{w 1}\right)$. These roughness values are comparable to the capillary-wave roughness of about $2.5 \AA$ for the water-gas interface measured with the same instrumental resolution. ${ }^{73,74}$ Finally, the main effect of compression in Region II $\left(A \rightarrow B ; A^{\prime} \rightarrow B^{\prime}\right)$ is to thicken the monolayer slightly, which is consistent with both close packing and microscopic compression. The fact that the product $A \times l_{1}$, which should be constant for a fixed amount of material, varies by about $4 \%$ between scans $\left\{A, A^{\prime}\right\}$ and $\left\{B, B^{\prime}\right\}$ is probably a measure of confidence one can have in this very simple model. These results establish that in Region II the PBLG molecules form a stable monomolecular layer on 
water with their rod axes parallel to the surface.

By contrast, both monodisperse and polydisperse PBLG films in Region IV are no longer monolayers. If the XR results are taken at face value, the conclusion would be that the relaxed film in this region is a bilayer characterized by a low-density second layer on top of a dense first layer [Fig. 5(c)]. In fact, as already mentioned, there is evidence that the film is not homogeneous in this region, and the proper quantitative interpretation of the best fit density profile is not clear. On the other hand, the $q_{z}$ positions of the minima and maxima of the $R / R_{F}$ curves depend only on the film thickness and not on the average density. The fact that the reflectivity can be well fit by the double-layer box model does indicate that the thickness of this second layer obtained from the best fits is reliable.

Based on the parameters listed in Table I(b), the following observations can be made: First, the thickness $\left(l_{1}\right.$ or $\left.l_{2}\right)$ of each of the two layers is comparable to the PBLG rod diameter of $\sim 13 \AA$. This strongly suggests that the PBLG molecules in the bilayer are confined within one or the other of the two layers and are still oriented parallel to the surface. Secondly, for all the bilayers measured in Region IV, the magnitude of the second layer densities $\left(\phi_{2}\right)$ was found to be only $30 \%-50 \%$ of the first layer densities $\left(\phi_{1}\right)$. This implies that on the average a smaller number of PBLG molecules occupy the top layer than the bottom layer. It is possible that the reflectivity arises from only some fraction of the film that is a homogeneous bilayer. The remainder could be a monolayer, but it is also possible that a sizeable fraction of the film consists of thicker aggregates that do not reflect. Thirdly, $\phi_{1}$ for the bilayers in Region IV is about $15 \%$ greater than the monolayer densities in Region II. All of these observations together suggest that as the film is compressed through Region III into IV, some of the PBLG molecules are pushed up from the monolayer into the second layer above, with others possibly pushed into even thicker aggregates. At the same time the first layer becomes slightly more packed than the monolayer in Region II. Moreover, throughout this process, the PBLG rods remain parallel to the interfacial plane.

There are a few other points to be made regarding the bilayers in Region IV. First, $l_{2}$ is slightly larger (by 1 to $2 \AA$ ) than $l_{1}$. Moreover, the roughness $\sigma_{2 g}$ for the second layergas interface is greater than those for the other two interfaces by more than $1 \AA$, giving a more diffuse appearance to the second layer-gas interface in the density profile. Considering that the molecules in the second layer have been pushed out of the monolayer upon compression, it may be possible that not all the PBLG rods in the second layer are located at the same height and some may not be oriented perfectly parallel to the interface. It is also important to note that the less dense second layer may be more susceptible to lateral density fluctuations over the surface. All of these points imply that the bilayer in Region IV is more likely to be microscopically inhomogeneous than the monolayer in Region II. This issue is considered more quantitatively in the XOSDS section below.

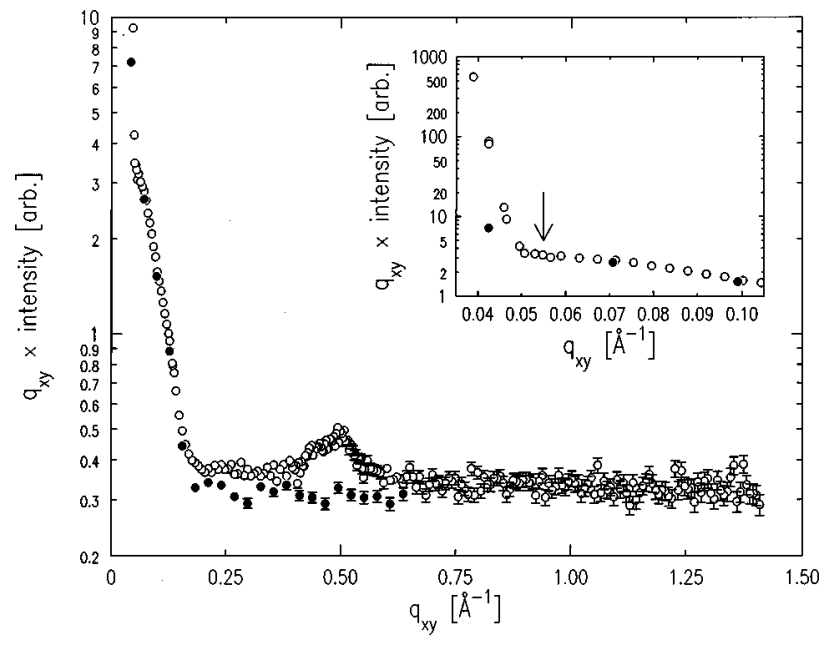

FIG. 6. The open circles $(O)$ show typical wide-range GID data from a PBLG film. This particular scan was measured on a monodisperse PBLG film at $(A,\langle\Pi\rangle)=\left(11.2 \AA^{2} /\right.$ monomer, $\left.9.8 \mathrm{dyn} / \mathrm{cm}\right)$. The filled circles $(\mathbf{O})$ show a background scan. The inset is an enlargement of a small $q_{x y}$ portion of the same data, shown with a different vertical scale. The arrow $(\downarrow)$ at $q_{x y}=0.055 \AA^{-1}$ indicates where a GID peak would be found if monodisperse PBLG molecules (rod length $\sim 115 \AA$ ) were to form smectic layers within the film. In the figures, the detected signal (apart from the structure near $q_{x y}=0.5 \AA^{-1}$ ) is dominated by diffuse scattering from the surface for $q_{x y}<\sim 0.048 \AA^{-1}$; small-angle scattering from the Kapton windows of the sample cell for $\sim 0.048 \AA^{-1}<q_{x y}<0.15 \AA^{-1}$; and scattering by gas above the surface for $q_{x y}>0.15 \AA^{-1}$.

\section{Grazing incidence diffraction (GID)}

Figure 6 is representative of wide GID scans taken on PBLG films. In order to account for the variation in the length of the beam path (i.e., the incident and totally reflected beam) viewed by the detector, the plotted quantity is $q_{x y} \times$ intensity. For most values of $q_{x y}$, the detected signal is attributed almost entirely to the background (filled circles). However, scattering from the film shows a structure around $q_{x y} \sim 0.5 \AA^{-1}$ that clearly rises above the background. Its $q_{x y}$ position corresponds to a characteristic lateral distance of $d$ $\sim 13 \AA$, which is close to the nearest-neighbor distance of 12.6 ̊ observed in the bulk smectic phase of PBLG. ${ }^{61}$ This repeat distance is comparable to the rod diameter of the molecule, and its presence indicates parallel alignment of PBLG helices within the LM plane. Rotation of the film about the surface normal showed no indication of variations in the peak intensities, i.e., the scattering from the illuminated sample area viewed by the detector (on the order of $1 \mathrm{~mm} \times 40 \mathrm{~mm}$ at $q_{x y} \sim 0.5 \AA^{-1}$ ) corresponded to a powder average with no evidence for large oriented domains. ${ }^{75}$

No other diffraction peaks were observed during the GID experiment. In particular, no evidence was found for smectic layering in the monodisperse PBLG films. Given the molecular length of about $115 \AA$ for the monodisperse PBLG ${ }^{61}$ the presence of one-dimensional smectic layers within the 2D plane would be signaled by a peak at $q_{x y}$ $\sim 0.055 \AA^{-1}$. No such peak was found in the GID data, as indicated by the arrow in the inset to Fig. 6 . However, there is a small possibility that a smectic peak was obscured by relatively high background in the small $q_{x y}$ region. Although this cannot be absolutely ruled out, the above result seems to 

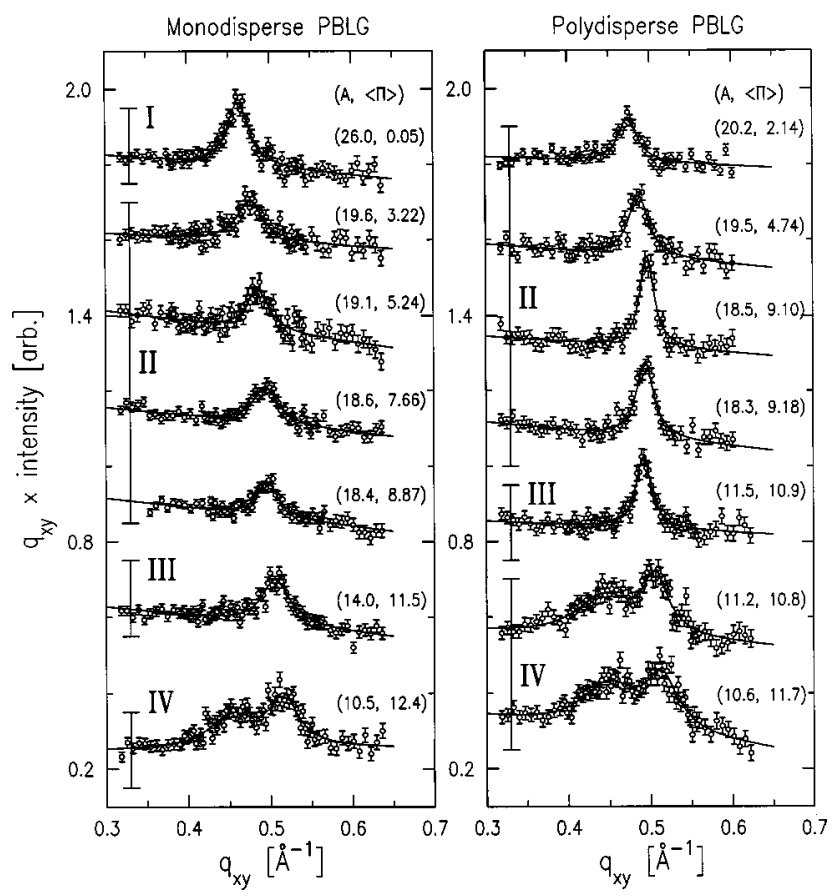

FIG. 7. Representative interhelix GID peaks from different regions in the $\Pi-A$ isotherms, for both monodisperse (left) and polydisperse (right) PBLG films. Area/monomer $\left(A\right.$, in $\left.\AA^{2}\right)$ and surface pressure $(\Pi$, in dyn $/ \mathrm{cm})$ values for the individual scans are indicated on the right side in each figure. The solid curves (-) are best fits to one (Regions I-III) or two (Region IV) Lorentzians with constant and linear background terms.

suggest that if there exists monodispersity-induced smectic order as observed in $3 \mathrm{D},{ }^{61}$ the extent of such ordering must be small in the PBLG LM.

Representative interhelix peaks around $q_{x y} \sim 0.5 \AA^{-1}$ from different regions in the $\Pi-A$ isotherms are shown in Fig. 7 for both mono- and polydisperse samples. The same qualitative behavior is observed regardless of sample dispersity. A peak is already present in Region I, without external pressure. In Region II, the peak shifts to larger $q_{x y}$ with increasing П. In the plateau region (III), little or no shift is observed. Finally, on the denser side of coexistence in Region IV, a weaker and broader second peak appears at slightly smaller $q_{x y}$. Quantitative analysis of these peaks was done by fitting the observed GID patterns to one (Regions I-III) or two (Region IV) Lorentzians with constant and linear background terms. Best fits are shown as solid lines in Fig. 7. Integrated intensities (area under the peak) and peak widths do not follow any consistent trend as a function of $A$ or $\Pi$. However, the peak positions $q_{x y}=q_{0}$, and therefore, the interhelix distances $d=2 \pi / q_{0}$ $=\lambda /\left[2 \sin \left(\theta_{0}\right)\right]$, display a distinct behavior in each of the four regions that is quantitatively similar for both mono- and polydisperse PBLG films.

Figures 8 and 9 summarize the dependence of the interhelix $d$-spacing on both $A$ and $\Pi$, obtained from measurements on two separate monodisperse PBLG films. Figure 8(b) shows the Region II part of Fig. 8(a). The compressionexpansion sequence used for each film is as follows: Film-1 was compressed first up to $A=18.8 \AA^{2} /$ monomer (open circles), expanded back to $20.2 \AA^{2} /$ monomer (filled circles),

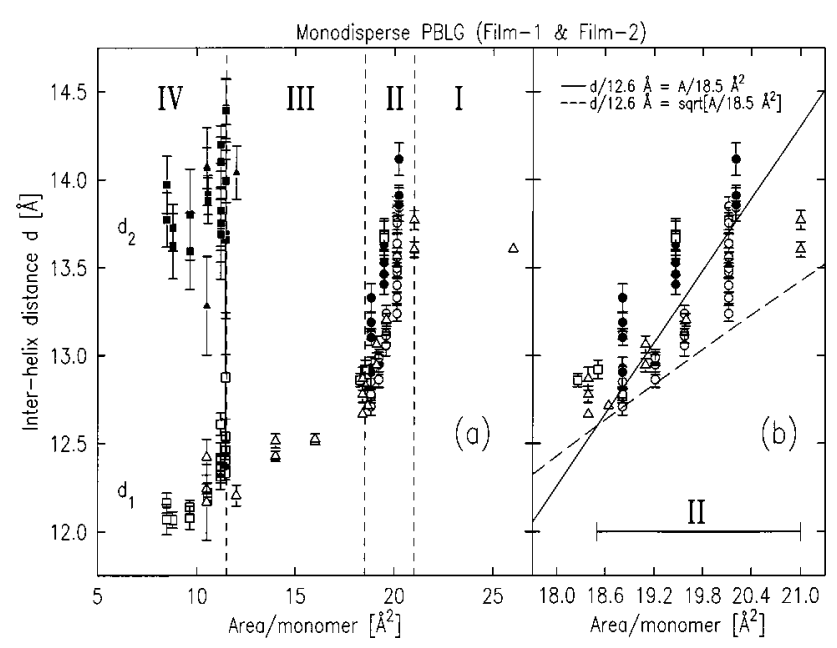

FIG. 8. (a) Interhelix $d$-spacing as a function of area/monomer, obtained from GID scans on two separate monodisperse PBLG films (Film-1 and Film-2). In Region IV,$d_{1}$ and $d_{2}$ indicate the two sets of $d$-spacings corresponding to the two GID peaks observed from bilayers. The data from Film-1 are separated into the first compression sequence $(\bigcirc, A$ $\geqslant 18.8 \AA^{2} /$ monomer $)$, the expansion sequence $\left(\bullet, 18.8 \leqslant A \leqslant 20.2 \AA^{2} /\right.$ monomer), and the second compression sequence ( $\square$ for $d_{1}, \square$ for $d_{2}$ ). The data for Film-2, which was compressed without intermediate expansion, are indicated by triangles ( $\triangle$ for $d_{1}, \boldsymbol{\Delta}$ for $d_{2}$ ). (b) An enlargement of Region II part of (a). The solid line $(-)$ and the dashed curve (---) describe a linear $(A \propto d)$ and a quadratic $\left(A \propto d^{2}\right)$ relationship between area/monomer and interhelix $d$-spacing, respectively, where each curve was assumed to go through the point $(A, d)=\left(18.5 \AA^{2} /\right.$ monomer, $\left.12.6 \AA\right)$.

and then compressed across Region III into IV (squares). Film-2 was compressed from Regions I-IV without intermediate expansion (triangles). At a given fixed $A$, two or more GID scans were typically taken, and the interhelix distances obtained from all the scans are presented together in Figs. 8 and 9. A PBLG monolayer (Regions I and II) is characterized by a single $d$-spacing, while a bilayer (Region IV) is

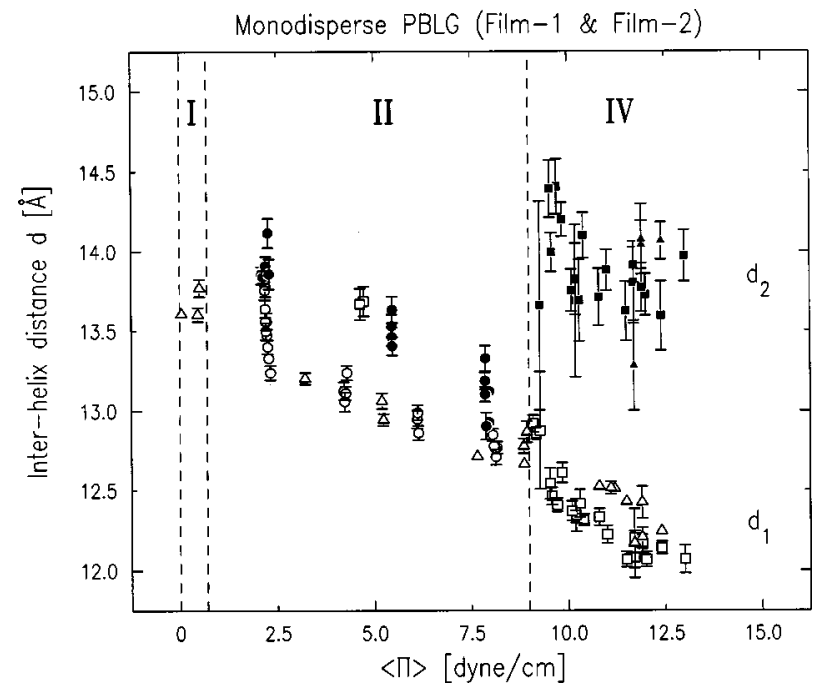

FIG. 9. Interhelix $d$-spacing as a function of surface pressure, obtained from GID scans on two separate monodisperse PBLG films (Film-1 and Film-2). The data from Film-1 are separated into the first compression sequence $(\bigcirc)$, the expansion sequence $(\bullet)$, and the second compression sequence ( $\square$ for $d_{1}, \boldsymbol{\square}$ for $d_{2}$ ). The data for Film-2 are indicated by triangles ( $\triangle$ for $d_{1}, \boldsymbol{\Delta}$ for $d_{2}$ ). 
associated with two $d$-spacings. The latter correspond to the two GID peaks observed and are labeled as $d_{1}$ (the first peak) and $d_{2}$ (the second peak at smaller $q_{x y}$ ).

The observation of a well defined interhelix distance in Region I $(\Pi=0)$ indicates that after being spread on water, PBLG molecules spontaneously aggregate and align themselves with their near neighbors to form $2 \mathrm{D}$ islands. As shown in Fig. 8(a), the $d$-spacing in this region remains almost unchanged at $d \sim 13.6 \AA$. This is consistent with the earlier suggestion that due to an incomplete surface coverage, macroscopic compression does not lead to microscopic compression in Region I. The interhelix distance here is slightly larger than the value of $d=12.6 \AA$ observed in the 3D smectic phase. ${ }^{61}$ In addition to the absence of external pressure in Region I, this could be due to the smaller number of nearest neighbors in $2 \mathrm{D}$.

In Region II ( $\Pi>0)$, macroscopic compression does result in a microscopic reduction of the interhelix distance, with the smallest $d$-spacing of $d \sim 12.6 \AA$ occurring at the Region II/III boundary. This is consistent with both a macroscopically full surface coverage expected for a closely packed LM (BAM) and a small increase in monolayer thickness $l_{1}$ with compression in Region II (XR). In Fig. 8(b), the two lines represent two extreme cases for a possible compression mode, assuming that they go through the point $(A, d)=\left(18.5 \AA^{2} /\right.$ monomer, $\left.12.6 \AA\right)$. The solid line assumes a linear relationship between $d$ and $A$, such that compression only reduces the interhelix distance perpendicular to the long axes of the helices; the dashed line assumes isotropic compression, i.e., $d \propto \sqrt{A}$. Unfortunately, the slopes for these two possibilities seem to bracket the data, and it is not possible to distinguish between them. Nevertheless, the molecules appear to remain confined to a monolayer throughout Region II. As for the $\Pi$ dependence, using the data points from the first compression sequence (open circles and triangles in Fig. $9)$, the average in-plane interhelix compressibility is estimated to be $\chi=-d^{-1}(\Delta d / \Delta \Pi) \sim 8 \times 10^{-3} \mathrm{~cm} / \mathrm{dyn}$ in Region II.

The $\Pi-A$ isotherm in Region II shows only small relaxation for $\Pi$ and is reversible with little hysteresis. However, note that the $d$ vs $A$ and $d$ vs $\Pi$ curves are not perfectly reversible in this region. Figures 8(b) and 9 show that expansion (filled circles) leads to relaxation of the $d$-spacing to values larger than those during the initial compression. On the other hand, the second compression points (open squares) appear to follow the expansion curve, indicating a smaller hysteresis in the expansion-recompression cycle than in the first compression-expansion cycle. Relaxation effects can also be seen in the time dependence. Figure 10 shows a gradual increase in the $d$-spacing with time (after the trough barrier was stopped) at two fixed values of $A$ in Region II. One possible explanation for these observations may be that the monolayer initially compressed to Region II contains many "defects" at the intermolecular level. For example, if there were defects such as holes between the ends of helices or between misaligned domains, the lateral interhelix compression necessary to attain a given macroscopic area would be greater than in the absence of holes. If this were so, then the relaxation of the interhelix $d$-spacing would correspond

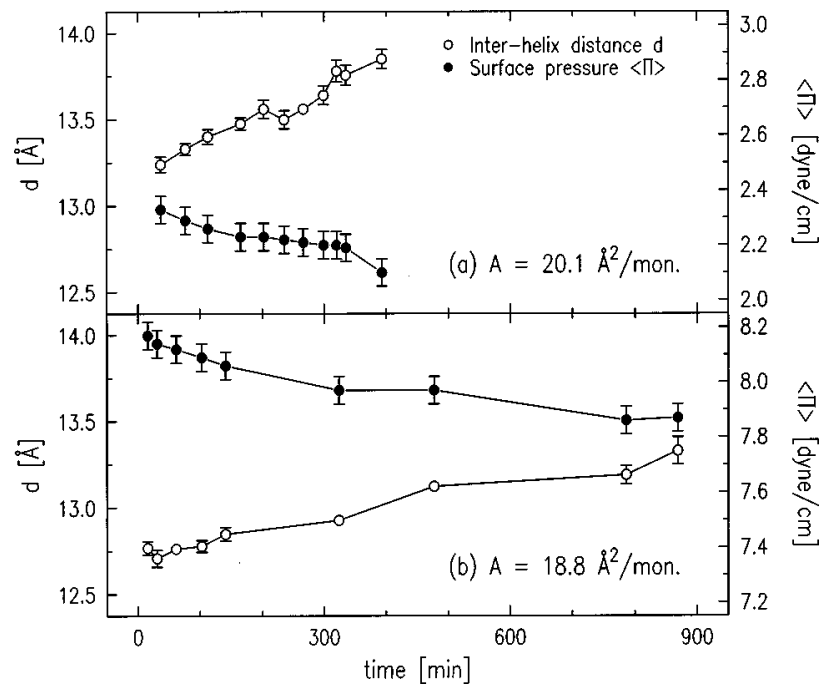

FIG. 10. Interhelix $d$-spacing $(\bigcirc)$ and surface pressure $(\bigcirc)$ as a function of time after the end of compression at $A=$ (a) $20.1 \AA^{2} /$ monomer and (b) 18.8 $\AA^{2} /$ monomer, obtained from measurements on a monodisperse PBLG film (Film-1) during the first compression sequence in Region II.

to the molecules having rearranged their positions to fill in some of these gaps. Alternatively, it is also possible that with time some molecules move out of the monolayer.

In Region III, a single interhelix distance $d_{1}$ (i.e., a monolayer) is observed for the most part, except near the Region III/IV boundary where the second $d$-spacing $d_{2}$ appears (signifying a bilayer). However, unlike in Region II, compression across Region III leaves the value of $d_{1}$ more or less unchanged at $d_{1} \sim 12.5 \AA$ [see Fig. 8(a)]. This suggests that the monolayer part of the coexisting phases in Region III remains similar to the compressed monolayer at the highest $\Pi$ in Region II. The fact that $d_{2}$ is not observed until near the Region III/IV boundary can be explained from a combination of the experimental geometry and the nature of bilayer formation. The footprint of the incident beam samples a surface area that is closer to the stationary edge of the trough (opposite to the movable barrier) than the barrier position at the beginning of coexistence. Assuming that the second layer preferably starts forming at the moving barrier and grows laterally with twice the barrier speed, the bilayer should enter the illuminated region at $A \sim 12-12.5 \AA^{2} /$ monomer, just before reaching Region IV. In order to investigate this behavior, specular reflectivity was monitored at constant $q_{z}$ during compression across Region III. A sudden change was always observed around areas of $A=11.5-13 \AA^{2} /$ monomer that signified the entrance of a bilayer region into the illuminated area. This also agrees with the BAM results suggesting that the second layer formation does not initiate everywhere but grows from near the barrier. Probably the moving barrier induces local time-dependent stresses that do not propagate down the length of the film.

As regards the two distinct $d$-spacings clearly observed for the PBLG bilayer in Region IV, the most likely explanation of their origin is an incommensurate structure in which interhelix distances are different and uncorrelated between the two layers of the bilayer. The XR results have shown that the average of the newly formed second layer is less dense 
than the first layer below. Moreover, the second GID peak corresponding to $d_{2}$ was always weaker than the first peak. These observations suggest that the second $d$-spacing $d_{2}$ corresponds to the interhelix distance in the second layer, while $d_{1}$ comes from the original monolayer underneath. This inference is further supported by the fact that during compression in Region IV,$d_{2}$ remains (within the large scatter in the data) close to the value $d=13.6 \AA$ observed for the uncompressed monolayer in Region I [see Figs. 8(a) and 9]. Since the second layer is not nearly as tightly packed as the first layer below, the aggregation of nearest-neighbor molecules in the second layer is probably similar to the behavior observed for the molecules in Region I. On the other hand, compression in Region IV leads to a systematic decrease in $d_{1}$ for the underlying monolayer. In fact, the $\Pi$ dependence of $d_{1}$ looks like an extrapolation of the behavior observed for the monolayer in Region II. This suggests that once the second layer is occupied to a certain degree, further upward transfer of molecules from the first layer becomes greatly inhibited. Consequently, compression of the bilayer results in a reduction of $d_{1}$ in the close packed first layer to values smaller than the limit of $d \sim 12.6 \AA$ that can be sustained by the monolayer in Region II.

Analysis of observed interhelix peaks also provides information about the extent of lateral positional correlations within the film, perpendicular to the PBLG rods. As mentioned already, the peak widths did not show any general trend with $A$ or $\Pi$. The FWHM of the interhelix $d_{1}$ peaks (i.e., for monolayers and the first layer of bilayers) ranged from $\Delta q_{x y} \sim 0.025$ to $0.06 \AA^{-1}$ with an average of $\Delta q_{x y}$ $\sim 0.04 \AA^{-1}$, while the FWHM for the $d_{2}$ peaks (i.e., for the second layer of bilayers) ranged from $\Delta q_{x y} \sim 0.05-0.1 \AA^{-1}$ with an average of $\Delta q_{x y} \sim 0.075 \AA^{-1}$. These observed widths are clearly greater than the experimental resolution of $\delta q_{x y} \sim 0.012 \AA^{-1}$. Assuming that the FWHM of the peaks arise from the convolution of a Lorentzian experimental resolution and Lorentzian broadening due to a finite lateral correlation length $\xi_{\perp}$ perpendicular to the helical axes of aligned PBLG molecules, the following relation can be obtained:

$$
\frac{\xi_{\perp}}{d}=\frac{q_{0}}{\pi\left[\Delta q_{x y}-\delta q_{x y}\right]},
$$

where the peak center is given by $q_{0}=2 \pi / d$. Using Eq. (11), the correlation length $\xi_{\perp}$ can be estimated to be only on the order of 3-12 interhelix distances for the PBLG rods in the monolayer and a few interhelix distances for those within the second layer of bilayers. Therefore, despite the tendency of PBLG molecules to align with their near neighbors, their lateral positional correlations do not extend very far, implying only short-range order. This result indicates that after being spread on water, PBLG molecules aggregate into a 2D glassy phase with only local positional ordering. Moreover, the lack of any systematic variation in $\xi_{\perp}$ with $A$ or $\Pi$ suggests that the local order remains "frozen in" during subsequent compression.

One interesting observation is that the interhelix correlation length $\xi_{\perp}$ is (and stays) comparable to the linear dimensions of the PBLG molecules used in this study $(\sim 100$

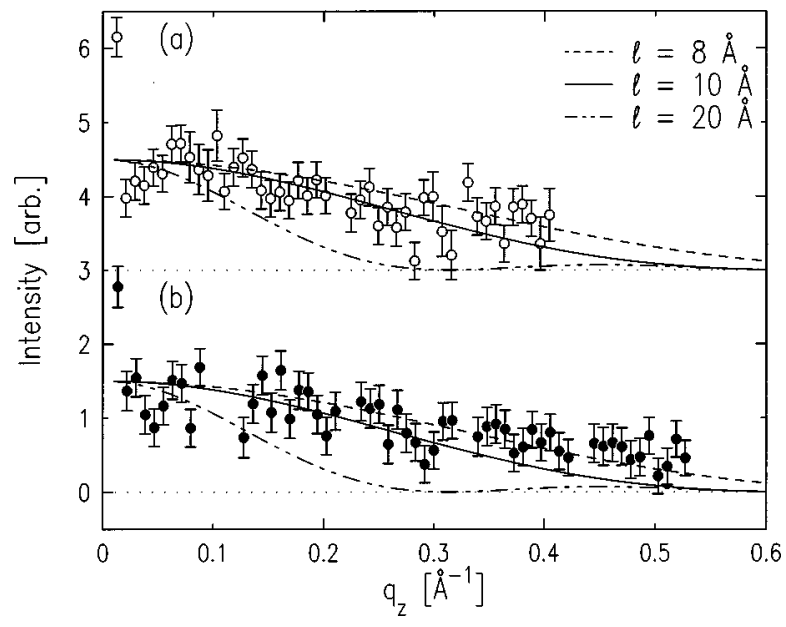

FIG. 11. Bragg rod along $q_{z}$ for the interhelix GID peak at $q_{x y}=2 \pi / d_{1}$, measured on a monodisperse PBLG film at (a) $A=19.2 \AA^{2} /$ monomer, $q_{x y}$ $=0.488 \AA^{-1} \quad\left(\bigcirc\right.$, Region II), and (b) $A=10.5 \AA^{2} /$ monomer, $q_{x y}$ $=0.502 \AA^{-1}(\bullet$, Region IV $)$. In each, the first data point near $q_{z}=0$ corresponds to the surface enhancement peak (Yoneda peak) at $\beta=\alpha_{c}$. The lines are model Bragg rods given by Eq. (12) for $l=8 \AA(--), 10 \AA(-)$, and $20 \AA(---)$, where $l$ is an effective "thickness" of the part of laterally correlated domains that gives rise to the GID peak.

$\AA$ ). Although the above GID results do not provide any information about the extent of orientational correlations, this observation seems to suggest that a typical size of an aligned domain may be on the order of the molecular length in both directions (parallel and perpendicular to the rod axes). If a PBLG monolayer consists of many such small domains in random orientations, extensive smectic order is not likely to be present even within a monodisperse film. This is consistent with the absence of a smectic layering peak in the GID data.

The absence of smecticlike order in the monodisperse PBLG films studied here is probably due to the strong aggregation tendency of PBLG. In the bulk study of monodisperse PBLG by Yu et al., the use of a small amount of trifluoroacetic acid (TFA) in a chloroform solution seemed to play an important role in achieving smectic ordering, presumably by inhibiting the aggregation of PBLG molecules. ${ }^{61,64}$ On the other hand, in the present study of monodisperse LMs on water, the evaporation of spreading solvent (3\%-TFA/97\%chloroform) is essentially instantaneous after film deposition. In a solvent-free environment, the formation of a $2 \mathrm{D}$ smectic phase (assuming it is possible) is likely to be suppressed by the local aggregation of PBLG molecules that results in glassy 2D domains.

Finally, in order to obtain additional evidence for the incommensurate structure of the PBLG bilayer, the $q_{z}$-dependence of the $d_{1}$ peaks was probed by rod scans. The Bragg-rods from the $d_{2}$ peaks could not be measured due to the lack of sufficient intensity. Figures 11(a) and 11(b) show the background subtracted Bragg-rod data on the $d_{1}$ peak (taken at fixed $q_{x y}=2 \pi / d_{1}$ ) from Regions II and IV, respectively. The lines in the figure are based on a model in which the electron density along the surface normal of a laterally correlated domain is simply approximated by a box of thickness $l$, and are described by 

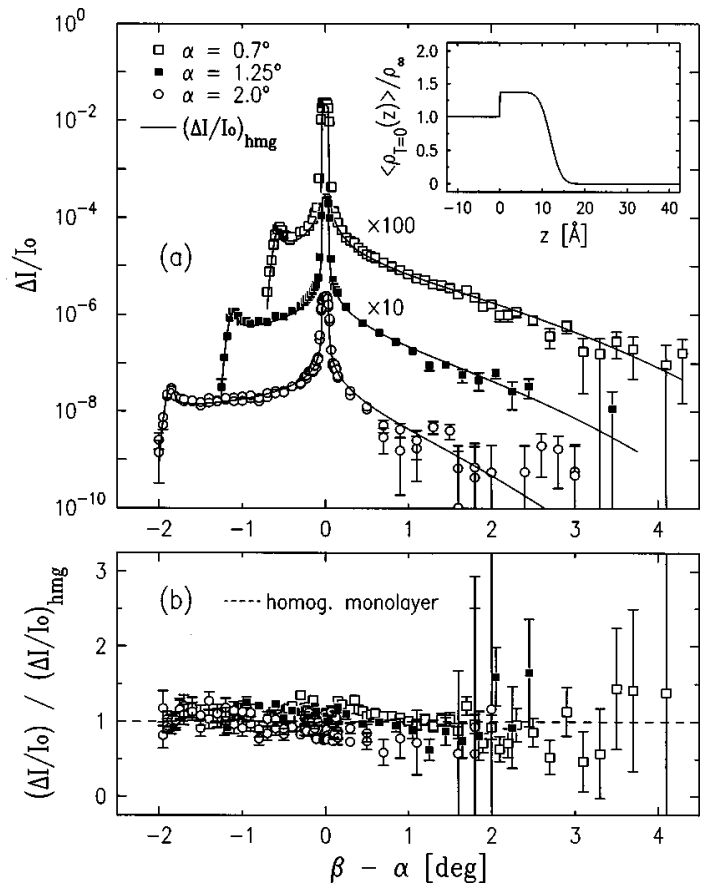

FIG. 12. (a) $\beta$-scans at fixed $\alpha$ from a polydisperse PBLG monolayer at $(A,\langle\Pi\rangle)=\left(19.2 \AA^{2} /\right.$ monomer, $\left.7.8 \mathrm{dyn} / \mathrm{cm}\right)$, shown as normalized intensity difference $\Delta I / I_{0}$ vs $\beta-\alpha$, where $\Delta I \equiv I(2 \theta=0)-(1 / 2)\left[I\left(+0.3^{\circ}\right)+I\right.$ $\left.\left(-0.3^{\circ}\right)\right]$. The solid curves $\left[\Delta I(\alpha, \beta) / I_{0}\right]_{\mathrm{hmg}}(-)$ theoretically expected for a homogeneous monolayer are based on Eq. (6), detector resolutions, and the average local electron density profile $\left\langle\rho_{T=0}(z)\right\rangle / \rho_{\infty}$ shown in the inset. The profile $\left\langle\rho_{T=0}(z)\right\rangle / \rho_{\infty}$ for this monolayer was obtained from a singlelayer box model and the fitting of $\left[\Delta I(\beta=\alpha) / I_{0}\right]_{\mathrm{hmg}}$ to the observed specular reflectivity $R\left(q_{z}\right)$, shown by the dashed curve (---) for $B^{\prime}$ in Fig. 5(b). (b) The ratio of the data $\Delta I / I_{0}$ to the solid line (-), i.e., calculated homogeneous contributions $\left[\Delta I / I_{0}\right]_{\mathrm{hmg}}$, in (a). The ratio should be unity (---) for a homogeneous PBLG monolayer.

$$
I_{\mathrm{rod}}\left(q_{z}\right) \propto\left[\frac{\sin \left(q_{z} l / 2\right)}{q_{z} l / 2}\right]^{2} .
$$

The comparison between the data and the model curves in Fig. 11 shows that whether the PBLG film is a monolayer or a bilayer, the part of laterally correlated domains with interhelix distance $d_{1}$ is confined effectively to a thickness of $l$ $=8-10 \AA$. This thickness is comparable to but slightly smaller than the total thickness of a PBLG monolayer observed by XR. Therefore, the rod-scan result implies that the $d_{1}$ interhelix peak originates from a single layer even in the case of a bilayer. This conclusion is also consistent with the interpretations given earlier, namely that in Region IV, the $d_{1}$ peaks arise only from the bottom layer, and the lateral order is uncorrelated between the two layers of the bilayer.

\section{Small-angle off-specular diffuse scattering (XOSDS)}

The results of $\beta$-scans on polydisperse PBLG films, taken at various fixed incident angles $\alpha$, are summarized in Fig. 12(a) for a monolayer in Region II and in Fig. 13(a) for an incomplete bilayer in Region IV. These data are from monolayer $B^{\prime}$ and bilayer $C^{\prime}$, respectively, whose $R / R_{F}$ data have already been shown in Fig. 5(b). In Figs. 12(a) and 13(a), the central peaks at $\beta-\alpha=0$ correspond to specular reflection, and the much smaller peaks at $\beta=\alpha_{c}$ on the left
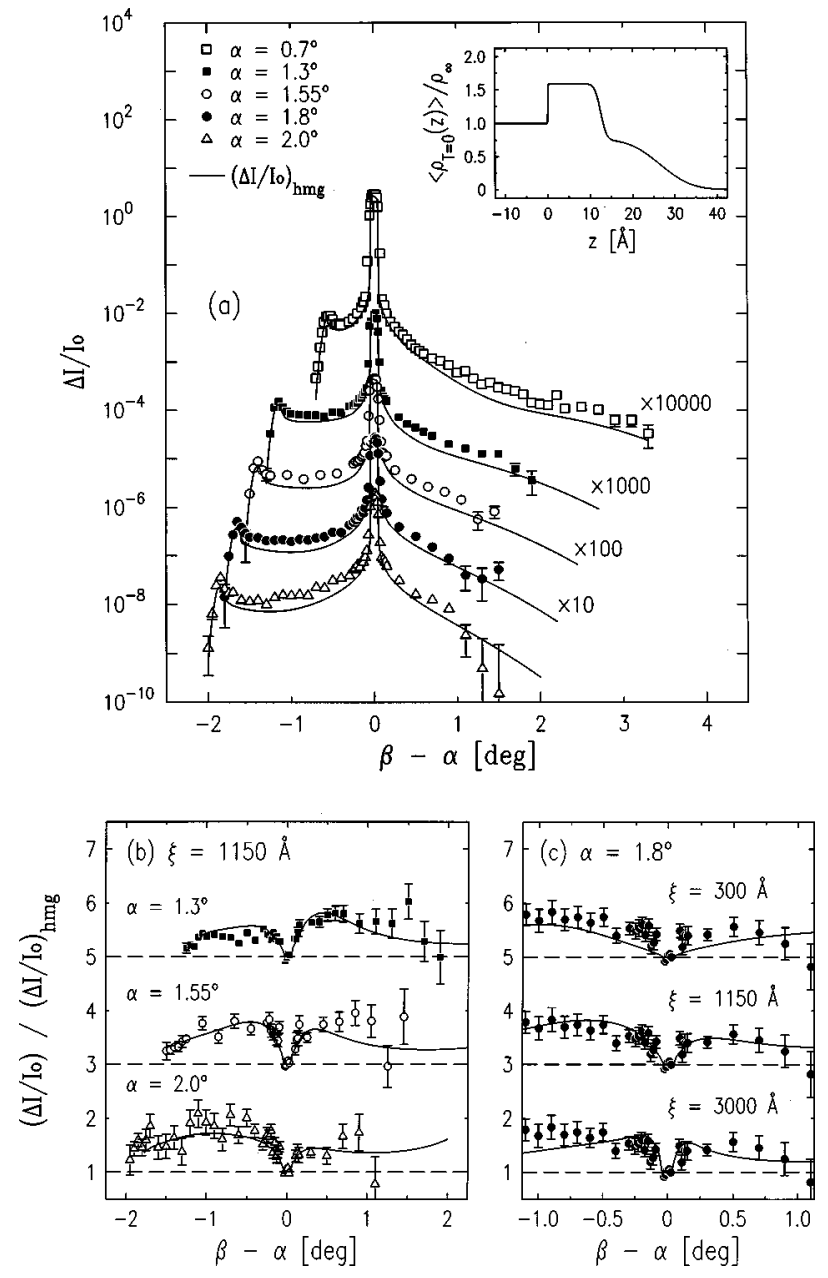

FIG. 13. (a) $\beta$-scans at fixed $\alpha$ from a polydisperse PBLG bilayer at $(A,\langle\Pi\rangle)=\left(9.7 \AA^{2} /\right.$ monomer, $\left.8.8 \mathrm{dyn} / \mathrm{cm}\right)$, shown as normalized intensity difference $\Delta I / I_{0}$ vs $\beta-\alpha$. The solid curves $\left[\Delta I(\alpha, \beta) / I_{0}\right]_{\mathrm{hmg}}(-)$ theoretically expected for a homogeneous bilayer are based on Eq. (6), detector resolutions, and the average local electron density profile $\left\langle\rho_{T=0}(z)\right\rangle / \rho_{\infty}$ shown in the inset. The profile $\left\langle\rho_{T=0}(z)\right\rangle / \rho_{\infty}$ for this bilayer was obtained from a double-layer box model and the fitting of $\left[\Delta I(\beta=\alpha) / I_{0}\right]_{\mathrm{hmg}}$ to the observed specular reflectivity $R\left(q_{z}\right)$, shown by the dashed curve (---) for $C^{\prime}$ in Fig. 5(b). (b) and (c) The ratio of the data $\Delta I / I_{0}$ to the solid line (-), i.e., calculated homogeneous contributions $\left[\Delta I / I_{0}\right]_{\mathrm{hmg}}$ in (a). The ratio would be unity (---) for a homogeneous PBLG bilayer. The solid lines $(-)$ in (b) are fits based on an inhomogeneous model Eq. (16) with noncapillary fluctuations of second layer-gas interfacial heights and correspond to a roughness of $\sigma_{2}=2.2 \AA$ and a correlation length of $\xi=1150 \AA$ along the interface. The solid lines (-) in (c) are the $\Delta I / I_{0}$ ratios calculated for three different values of $\xi$ at $\alpha=1.8^{\circ}$ and $\sigma_{2}=2.2 \AA$.

wings are the Yoneda or surface enhancement peaks, which originate from the Fresnel transmission factor $T_{F}(\beta)$.

The solid curves in Figs. 12(a) and 13(a) correspond to the calculated normalized intensity difference $[\Delta I(\alpha, \beta) /$ $\left.I_{0}\right]_{\text {hmg }}$ expected for homogeneous films. The calculation is based on the known temperature and surface tension of each PBLG film and the slit-defined resolution functions, as explained in Sec. IIC3. The layering structure factor $\Phi_{0}\left(q_{z}\right)$ for each film was obtained by using a box model for the average local density profile $\left\langle\rho_{T=0}(z)\right\rangle$ and fitting $\left[\Delta I(\alpha, \beta=\alpha) / I_{0}\right]_{\mathrm{hmg}}$ to the measured specular reflectivity $R\left(q_{z}\right)$. The best-fit $R / R_{F}$ curves obtained from this fitting procedure are shown as dashed lines in Fig. 5(b). The corre- 
sponding intrinsic density profiles $\left\langle\rho_{T=0}(z)\right\rangle$, shown in the insets of Figs. 12(a) and 13(a), are consistent with the total averages $\langle\rho(z)\rangle$ obtained in Sec. III B 1. In both the fitting of $\left[\Delta I(\alpha, \beta=\alpha) / I_{0}\right]_{\mathrm{hmg}}$ to $R\left(q_{z}\right)$ and the theoretical calculation of $\left[\Delta I(\alpha, \beta) / I_{0}\right]_{\mathrm{hmg}}$ for $\beta$-scans, the smallest wavelength for the capillary wave modes was assumed to be of the order of the PBLG rod diameter $d \sim 12.6 \AA$ and the upper cutoff wave vector value was taken to be $q_{\max } \sim 2 \pi / d=0.5 \AA^{-1}$.

As evident in Fig. 12(a), the agreement between the data $\Delta I(\alpha, \beta) / I_{0}$ and the theoretical curves $\left[\Delta I(\alpha, \beta) / I_{0}\right]_{\mathrm{hmg}}$ is very good for the PBLG monolayer in Region II. The ratios between the data and the theory are close to unity, as shown in Fig. 12(b). Since the theory is based on the assumption that surface scattering originates only from the capillary wave fluctuations with intensity modified by the average local density profile $\left\langle\rho_{T=0}(z)\right\rangle$, the good agreement implies that the monolayer is microscopically homogeneous. That is, (i) the height fluctuations of the water-monolayer and monolayer-gas interfaces are conformal with each other and consistent with the capillary wave model, and (ii) long wavelength density variations within the PBLG monolayer, if they exist, must be very small.

By contrast, Fig. 13(a) shows that the off-specular ( $\beta$ $-\alpha \neq 0)$ parts of the data for the incomplete bilayer in Region IV are consistently higher than the theoretical curves predicted for a homogeneous bilayer. Considering that the structure factor $\Phi_{0}\left(q_{z}\right)$ used in the homogeneous model is based on fits to the measured specular reflectivity, the agreement between data and model near the specular condition $(\beta-\alpha=0)$ is as expected. However, as clearly evident in Figs. 13(b) and 13(c), showing the same data normalized to the homogeneous model, the data exceeds the model by up to a factor of two in the off-specular regions. This additional scattering above the homogeneous prediction must originate from the term $\delta \rho_{T=0}\left(r_{x y}, z\right) \neq 0$ in Eq. (8). Therefore, the observation of off-specular excess scattering provides a measure of noncapillary lateral density inhomogeneities within the bilayer.

As already pointed out, the lower density in the second layer of the bilayer indicates that it is not densely packed, providing more room for (spatial) fluctuations in lateral density. The diffuseness of the second layer-gas interface evident in the average density profile for the bilayer [see the inset in Fig. 13(a)] is also suggestive of variations in the heights of that interface over the surface. These suggestions are supported further by the observation of relatively small differences $(\sim 10 \%)$ between the two interhelix distances $d_{1}$ and $d_{2}$ in the GID despite the significant differences in average density in the two layers. Therefore, it is reasonable to assume that surface inhomogeneities with $\delta \rho_{T=0}\left(r_{x y}, z\right) \neq 0$ are mostly concentrated in the second layer of the PBLG bilayer. Given that the molecules in the second layer have been pushed out upward from the monolayer below, the origin of second layer inhomogeneities might be a distribution in the heights of molecular centers or in the orientation of the molecular axes, with some of them being not perfectly parallel to the interface. Another possibility would be molecular density variations within the second layer, with some regions of the second layer being more densely occupied than others.
For example, such density variations would arise if the second layer consisted of microscopic islands on top of the first layer. It is difficult to distinguish between these possibilities from the obtained experimental data. Consequently, a quantitative analysis of the excess scattering has been carried out by assuming a simple model in which the second layer inhomogeneities are represented as noncapillary height fluctuations of the second layer-gas interface.

The model assumes that the local electron density within the second layer is constant at $\rho_{2}=\rho_{\infty} \cdot \phi_{2}$, but that the height $h_{2}\left(r_{x y}\right)+l_{2}+l_{1}$ of the second layer-gas interface relative to the position of the monolayer-water interface fluctuates spatially about $\left\langle h_{2}(0)\right\rangle=0$ over the surface. Since the range of $(\beta-\alpha)$ in the measured $\beta$-scans is not large enough to probe correlations on the length scale of molecular anisotropies, it is convenient to assume that the variations in $h_{2}\left(r_{x y}\right)$ are isotropic. Then, in the laboratory frame, the height variations of the second layer-gas interface can be described by the sum $\left\{h_{\text {tot }}\left(r_{x y}\right)\right\}=\left\{h\left(r_{x y}\right)\right\}+\left\{h_{2}\left(r_{x y}\right)\right\}$ of the capillary $\left\{h\left(r_{x y}\right)\right\}$ and the noncapillary $\left\{h_{2}\left(r_{x y}\right)\right\}$ height distributions. Assuming that $\left\{h\left(r_{x y}\right)\right\}$ and $\left\{h_{2}\left(r_{x y}\right)\right\}$ are statistically independent, the use of this model in Eq. (8) in the limit that the $h_{2}-h_{2}$ correlation function $c_{2}\left(r_{x y}\right)$ $\equiv\left\langle h_{2}\left(r_{x y}\right) h_{2}(0)\right\rangle \ll 1 / q_{z}^{2}$ leads to

$$
\begin{aligned}
\frac{1}{A_{0}}\left(\frac{d \sigma}{d \Omega}\right)_{\mathrm{inhmg}} \approx & \frac{1}{16 \pi^{2}}\left(\frac{q_{c}}{2}\right)^{4} \frac{\phi_{2}^{2} e^{-\sigma_{2}^{2} q_{z}^{2}}}{\sin (\alpha)} \\
& \times \int d^{2} \mathbf{r}_{x y} e^{-i \mathbf{q}_{x y} \cdot \mathbf{r}_{x y}} e^{-(1 / 2) g\left(r_{x y}\right) q_{z}^{2}} c_{2}\left(r_{x y}\right),
\end{aligned}
$$

where $\sigma_{2}^{2}=\left\langle h_{2}^{2}(0)\right\rangle$ is the mean-square roughness of the second layer-gas interface and $g\left(r_{x y}\right)=\left\langle\left[h\left(r_{x y}\right)-h(0)\right]^{2}\right\rangle$. Using the convolution theorem and the proper normalization of the capillary wave spectrum, ${ }^{69,70} \mathrm{Eq}$. (13) can be expressed as a convolution of the capillary $(h)$ and noncapillary $\left(h_{2}\right)$ height fluctuations in reciprocal space:

$$
\begin{aligned}
\frac{1}{A_{0}}\left(\frac{d \sigma}{d \Omega}\right)_{\text {inhmg }} \approx & \frac{1}{16 \pi^{2}}\left(\frac{q_{c}}{2}\right)^{4} \frac{\phi_{2}^{2} e^{-\sigma_{2}^{2} q_{z}^{2}}}{\sin (\alpha)} \\
& \times \frac{1}{(2 \pi)^{2}} \int_{q_{x y}^{\prime} \leqslant q_{\max }} d^{2} \mathbf{q}_{x y}^{\prime} \frac{2 \pi \eta}{q_{x y}^{\prime 2}}\left(\frac{q_{x y}^{\prime}}{q_{\max }}\right)^{\eta} \\
& \times C_{2}\left(\mathbf{q}_{x y}-\mathbf{q}_{x y}^{\prime}\right),
\end{aligned}
$$

where $\eta=\left(k_{B} T / 2 \pi \gamma\right) q_{z}^{2}$ as in the homogeneous case, and $C_{2}\left(\mathbf{q}_{x y}\right)$ is the 2D Fourier transform of $c_{2}\left(r_{x y}\right)$. For computational simplicity, an exponentially decaying $h_{2}-h_{2}$ correlation function

$$
c_{2}\left(r_{x y}\right)=\sigma_{2}^{2} e^{-r_{x y} / \xi},
$$

is assumed with a correlation length $\xi$, such that

$$
C_{2}\left(\mathbf{q}_{x y}\right)=\frac{2 \pi \sigma_{2}^{2} \xi^{2}}{\left[1+\xi^{2}\left|\mathbf{q}_{x y}\right|^{2}\right]^{3 / 2}} .
$$

With the substitution of Eq. (15b), Eq. (14) becomes 


$$
\begin{aligned}
\frac{1}{A_{0}}\left(\frac{d \sigma}{d \Omega}\right)_{\mathrm{inhmg}} \approx & \frac{1}{16 \pi^{2}}\left(\frac{q_{c}}{2}\right)^{4} \frac{4 \phi_{2}^{2} \sigma_{2}^{2} \xi^{2} e^{-\sigma_{2}^{2} q_{z}^{2}}}{q_{\max }^{\eta} \sin (\alpha)} \\
& \times \int_{0}^{q_{\max }^{\eta}} d t X\left(q_{x y}^{\prime}=t^{1 / \eta}\right),
\end{aligned}
$$

with $q_{x y}=\left|\mathbf{q}_{x y}\right|, q_{x y}^{\prime}=\left|\mathbf{q}_{x y}^{\prime}\right|$, and

$$
\begin{aligned}
X\left(q_{x y}^{\prime}\right)= & \frac{1}{\left[1+\xi^{2}\left(q_{x y}-q_{x y}^{\prime}\right)^{2}\right] \sqrt{1+\xi^{2}\left(q_{x y}+q_{x y}^{\prime}\right)^{2}}} \\
& \times E\left(\sqrt{\frac{4 \xi^{2} q_{x y} q_{x y}^{\prime}}{1+\xi^{2}\left(q_{x y}+q_{x y}^{\prime}\right)^{2}}}\right),
\end{aligned}
$$

where $E(k)$ is the complete elliptic integral of the second kind. The $q_{x y}^{\prime}$-integration in Eq. (16a) is done numerically.

In order to obtain a correlation length associated with second layer inhomogeneities, the numerical convolution of Eq. (16) with the experimental resolution function has been fit simultaneously to all of the measured excess scattering, $\left[\Delta I(\alpha, \beta) / I_{0}\right]_{\mathrm{inhmg}}=\Delta I(\alpha, \beta) / I_{0}-\left[\Delta I(\alpha, \beta) / I_{0}\right]_{\mathrm{hmg}}, \quad$ observed from the PBLG bilayer at various sets of $(\alpha, \beta)$. Only $\sigma_{2}$ and $\xi$ were allowed to vary in the fitting, while $q_{\max }$ $=0.5 \AA^{-1}$ was used as in the homogeneous case, and the relative electron density in the second layer was fixed at the XR-based value of $\phi_{2}=\rho_{2} / \rho_{\infty}=0.80$. All the other parameters are known. The best fit is obtained with $\xi=1150 \AA$ and $\sigma_{2}=2.2 \AA$, where the range of errors determined by $67 \%$ confidence limits are $400 \AA<\xi<3200 \AA$ and $1.7 \AA<\sigma_{2}$ $<3.1 \AA$. The normalized intensity ratios $[\Delta I(\alpha, \beta) /$ $\left.I_{0}\right] /\left[\Delta I(\alpha, \beta) / I_{0}\right]_{\mathrm{hmg}}$ calculated from the best fit are plotted as solid curves in Fig. 13(b) at various incident angles $\alpha$. The ratio is unity at $\beta=\alpha$ and increases above unity as $\beta$ moves away from $\alpha$. The inverse width of the "valley" centered at $\beta=\alpha$ is a measure of the correlation length $\xi$, as demonstrated in Fig. 13(c), in which the intensity ratios are plotted at $\alpha=1.8^{\circ}$ and $\sigma_{2}=2.2 \AA$ for three different values of $\xi$.

From the above analysis, one can estimate the correlation length associated with the second layer inhomogeneity to be on the order of $\xi \sim 1000 \AA$, which is about 80 times the rod diameter or about 7 to 8 times the rod length of typical PBLG molecules. For example, if the inhomogeneity arose from second layer islands on top of the monolayer, this would imply a mean island size of $\sim 1000 \AA$. Since the obtained correlation length is one order of magnitude greater than the molecular size, and since there is no reason to expect a critical behavior in this system, it is unlikely that the origin of the inhomogeneity can be attributed to local or intramolecular density variations, such as random configurations of side chains of PBLG helices. The above analysis cannot distinguish between other plausible possibilities such as lateral variations in molecular height, orientation, or packing density over the second layer. However, it does clearly show that the observation of excess scattering from the PBLG bilayer in Region IV is consistent with nonhomogeneity of the newly formed second layer. This result is to be contrasted from the case of compressed PBLG monolayers in Region II, for which all of the long-wavelength surface fluctuations can be attributed to capillary waves.

\section{SUMMARY}

The structures of both mono- and polydisperse PBLG Langmuir films on water have been studied using BAM and $\mathrm{x}$-ray scattering techniques. The observed microscopic behavior showed no significant dependence on sample dispersity. In particular, no evidence was found for the presence of smectic layers in monodisperse films. On the basis of the results presented, the following summarizes a model for the structural changes that the PBLG LM undergoes with decreasing $A$ :

(I) $A>\sim 21 \AA^{2} /$ monomer: As soon as being spread on water surface, PBLG rods lie down flat on water surface, self-aggregate laterally, and align themselves parallel to their immediate neighbors to form solidlike 2D islands. Compression in this regime $(\Pi=0)$ only reduces areas of bare water surface coexisting with monolayer islands and results in no structural changes at the intermolecular level.

(II) $\sim 18.5<A<\sim 21 \AA^{2} /$ monomer: The PBLG monolayer homogeneously covers the entire surface. Compression in this regime results in both a steep rise in surface pressure and a reduction of the interhelix distance between aligned PBLG rods from $\sim 13.6 \AA$ at $\Pi=0$ to $\sim 12.6 \AA$ at $\Pi$ $\sim 9 \mathrm{dyn} / \mathrm{cm}$. PBLG rods remain parallel to the interface during compression.

(III) $\sim 11.5<A<\sim 18.5 \AA^{2} /$ monomer: The PBLG monolayer can sustain surface pressures only up to a maximum of $\Pi \sim 9 \mathrm{dyn} / \mathrm{cm}$. Compression past this limit results in an upward transfer of PBLG molecules to a second layer. The bilayer formation is not uniform over the surface, but starts preferably near the moving barrier and grows in the compression direction. During compression through this coexistence region, the structure of the monolayer phase remains similar to that of a highly compressed monolayer in (II).

(IV) $A<\sim 11.5 \AA^{2} /$ monomer: The film is dominated by an incomplete, incommensurate, and inhomogeneous bilayer. There are sizable homogeneous fractions within the second layer, and these are less densely occupied than the close packed first layer. Within these fractions, the interhelix distance is larger than that of the underlying monolayer but is comparable to that observed for uncompressed monolayer islands in (I). The fact that the monolayer $d$-spacing continues to decrease with increasing $\Pi$ in this region, suggests that the presence of the second layer hinders a further upward transfer of PBLG molecules out of the first layer.

The analysis of interhelix GID peaks shows that for both mono- and bilayers, the extent of lateral positional correlations between aligned PBLG rods is limited to a range of a few to no more than 15 interhelix distances. This result on PBLG LM is one of the limited number of cases where a LM phase with only short-range positional order provided observable x-ray scattering. Moreover, it supports the recently made suggestion ${ }^{27}$ that the increased scattering power provided by molecules with a large number of electrons might enable $\mathrm{x}$-ray scattering studies of noncrystalline LM phases and phase transitions involving such phases.

One consequence of the use of large molecules, however, is stronger mutual attraction between them and, hence, their tendency to aggregate into a solid monolayer phase. 
Moreover, the results of this study suggest that if the solid LM phase formed is noncrystalline and involves large rodlike molecules, it tends to remain noncrystalline up to the highest surface pressure that can be sustained by the monolayer. This is probably because structural rearrangements required to transform the glassy phase into another $2 \mathrm{D}$ phase would include extensive molecular reorientations and are, therefore, more difficult to achieve with such large molecules confined in tightly packed spaces. One possibility for avoiding this type of aggregation behavior might be introduction of some repulsive forces between large molecules (e.g., through chemical modifications of side chains for helical polypeptides). If this can be achieved, $\mathrm{x}$-ray scattering studies of 2D phase transitions involving fluid LM phases may become possible. For $\alpha$-helical polypeptides, the reduction of intermolecular attraction is an important next step that might facilitate monodispersity-induced smectic layering in LMs.

\section{ACKNOWLEDGMENTS}

The authors thank H. Tostmann, O. G. Shpyrko, K. Penanen, E. DiMasi, B. M. Ocko, and M. Deutsch for helpful discussions regarding the off-specular diffuse scattering technique. The Harvard contribution to this work was supported by Grant No. NSF-DMR-98-72817. Work at the University of Massachusetts was supported by Grant No. NSF-DMR95-10031. The X-ray experiments were carried out on the Harvard/BNL liquid surface spectrometer at Beamline X22B in the National Synchrotron Light Source, Brookhaven National Laboratory. This facility is supported by No. DEAC02-76CH00016.

${ }^{1}$ C. M. Knobler, Adv. Chem. Phys. 77, 397 (1990).

${ }^{2}$ C. M. Knobler and R. C. Desai, Annu. Rev. Phys. Chem. 43, 207 (1992).

${ }^{3}$ H. Möhwald, Annu. Rev. Phys. Chem. 41, 441 (1990).

${ }^{4} \mathrm{~J}$. Als-Nielsen and H. Möhwald, in Handbook of Synchrotron Radiation, Vol. 4, edited by S. Ebashi, E. Rubenstein, and M. Koch (North Holland, Amsterdam, 1991), p. 1.

${ }^{5}$ J. Als-Nielsen, D. Jacquemain, K. Kjaer, F. Leveiller, M. Lahav, and L. Leiserowitz, Phys. Rep. 246, 251 (1994).

${ }^{6}$ P. S. Pershan, Structure of Liquid Crystal Phases (World Scientific, Singapore, 1988).

${ }^{7}$ J. D. Brock, R. J. Birgeneau, J. D. Litster, and A. Aharony, Phys. Today July, 52-59 (1989).

${ }^{8}$ O. M. Magnussen, B. M. Ocko, M. Deutsch, M. J. Regan, P. S. Pershan, D. Abernathy, G. Grubel, and J.-F. Legrand, Nature (London) 384, 250 (1996).

${ }^{9}$ M. Deutsch, O. M. Magnussen, B. M. Ocko, M. J. Regan, and P. S. Pershan, in Self-Assembled Monolayers of Thiols, edited by A. Ulman (Academic, San Diego, 1998).

${ }^{10}$ B. R. Malcolm, Nature (London) 195, 901 (1962).

${ }^{11}$ B. R. Malcolm, Polymer 7, 595 (1966).

${ }^{12}$ B. R. Malcolm, Proc. R. Soc. London, Ser. A 305, 363 (1968).

${ }^{13}$ G. I. Loeb, J. Colloid Interface Sci. 26, 236 (1968).

${ }^{14}$ G. I. Loeb and R. E. Baier, J. Colloid Interface Sci. 27, 38 (1968).

${ }^{15}$ B. R. Malcolm, J. Polym. Sci., Part C: Polym. Symp. 34, 87 (1971).

${ }^{16}$ D. W. Goupil and F. C. Goodrich, J. Colloid Interface Sci. 62, 142 (1977).

${ }^{17}$ D. G. Cornell, J. Colloid Interface Sci. 70, 167 (1979).

${ }^{18}$ T. Takenaka, K. Harada, and M. Matsumoto, J. Colloid Interface Sci. 73, 569 (1980)

${ }^{19}$ G. Gabrielli, P. Baglioni, and E. Ferroni, J. Colloid Interface Sci. 81, 139 (1981).

${ }^{20}$ F. Takeda, M. Matsumoto, T. Takenaka, and Y. Fujiyoshi, J. Colloid Interface Sci. 84, 220 (1981).

${ }^{21}$ F. Takeda, M. Matsumoto, T. Takenaka, Y. Fujiyoshi, and N. Uyeda, J. Colloid Interface Sci. 91, 267 (1983).

${ }^{22}$ B. R. Malcolm, J. Colloid Interface Sci. 104, 520 (1985).
${ }^{23}$ H. Motschmann, R. Reiter, R. Lawall, G. Duda, M. Stamm, G. Wegner, and W. Knoll, Langmuir 7, 2743 (1991).

${ }^{24}$ P. Lavigne, P. Tancrede, F. Lamarche, and J.-J. Max, Langmuir 8, 1988 (1992).

${ }^{25}$ P. Lavigne, P. Tancrede, F. Lamarche, M. Grandbois, and C. Salesse, Thin Solid Films 242, 229 (1994).

${ }^{26}$ D. Sohn, H. Yu, J. Nakamatsu, P. S. Russo, and W. H. Daly, J. Poly. Sci. B 34, 3025 (1996).

${ }^{27}$ M. Fukuto, K. Penanen, R. K. Heilmann, P. S. Pershan, and D. Vaknin, J. Chem. Phys. 107, 5531 (1997).

${ }^{28}$ I. Uematsu, Adv. Polym. Sci. 59, 37 (1984).

${ }^{29}$ J. Watanabe, Y. Fukuda, R. Gehani, and I. Uematsu, Macromolecules 17, 1004 (1984).

${ }^{30}$ J. Watanabe, H. Ono, I. Uematsu, and A. Abe, Macromolecules 18, 2141 (1985).

${ }^{31}$ J. Watanabe, M. Goto, and T. Nagase, Macromolecules 20, 298 (1987).

${ }^{32}$ E. Iizuka, K. Abe, K. Hanabusa, and H. Shirai, in Current Topics in Polymer Science, Vol. I (Hanser, Munich, 1987), pp. 235-248.

${ }^{33}$ M. Ballauff, Angew. Chem. Int. Ed. Engl. 28, 253 (1989).

${ }^{34}$ G. Duda, A. J. Schouten, T. Arndt, G. Lieser, G. F. Schmidt, C. Bubeck, and G. Wegner, Thin Solid Films 159, 221 (1988).

${ }^{35}$ S. Schwiegk, T. Vahlenkamp, G. Wegner, and Y. Xu, Thin Solid Films 210/211, 6 (1992).

${ }^{36}$ S. Schwiegk, T. Vahlenkamp, Y. Xu, and G. Wegner, Macromolecules 25, 2513 (1992).

${ }^{37}$ K. Mathauer, A. Schmidt, W. Knoll, and G. Wegner, Macromolecules 28, 1214 (1995)

${ }^{38}$ W. Hickel, G. Duda, M. Jurich, T. Krohl, K. Rochford, G. I. Stegeman, J. D. Swalen, G. Wegner, and W. Knoll, Langmuir 6, 1403 (1990).

${ }^{39}$ S. Lee, J. R. Dutcher, B. Hillebrands, G. I. Stegeman, W. Knoll, G. Duda, G. Wegner, and F. Nizzoli, Mater. Res. Soc. Symp. Proc. 188, 355 (1990).

${ }^{40}$ F. Nizzoli, B. Hillebrands, S. Lee, G. I. Stegeman, G. Duda, G. Wegner, and W. Knoll, Mater. Sci. Eng., B 5, 173 (1990).

${ }^{41}$ K. Mathauer, A. Mathy, C. Bubeck, G. Wegner, W. Hickel, and U. Scheunemann, Thin Solid Films 210/211, 449 (1992).

${ }^{42}$ A. Mathy, K. Mathauer, G. Wegner, and C. Bubeck, Thin Solid Films 215, 98 (1992).

${ }^{43}$ H. Menzel, B. Weichart, and M. L. Hallensleben, Thin Solid Films 223, 181 (1993).

${ }^{44}$ H. Menzel, M. L. Hallensleben, A. Schmidt, W. Knoll, T. Fischer, and J. Stumpe, Macromolecules 26, 3644 (1993).

${ }^{45}$ V. V. Tsukruk, M. D. Foster, D. H. Reneker, A. Schmidt, and W. Knoll, Langmuir 9, 3538 (1993).

${ }^{46}$ I. H. Musselman, D. L. Smith, E. P. Enriquez, V. F. Guarisco, and E. T. Samulski, J. Vac. Sci. Technol. A 12, 2523 (1994).

${ }^{47}$ A. Schmidt, K. Mathauer, G. Reiter, M. D. Foster, M. Stamm, G. Wegner, and W. Knoll, Langmuir 10, 3820 (1994).

${ }^{48}$ V. V. Tsukruk, M. D. Foster, D. H. Reneker, A. Schmidt, H. Wu, and W. Knoll, Macromolecules 27, 1274 (1994).

${ }^{49}$ T. R. Vierheller, M. D. Foster, A. Schmidt, K. Mathauer, W. Knoll, G. Wegner, S. Satija, and C. F. Majkrzak, Macromolecules 27, 6893 (1994).

${ }^{50} \mathrm{G}$. Wegner and K. Mathauer, Mater. Res. Soc. Symp. Proc. 247, 767 (1992).

${ }^{51}$ D. Neher, Adv. Mater. 7, 691 (1995).

${ }^{52}$ C. Robinson, Trans. Faraday Soc. 52, 571 (1956).

${ }^{53}$ C. Robinson and J. C. Ward, Nature (London) 180, 1183 (1957).

${ }^{54}$ C. Robinson, J. C. Ward, and R. B. Beevers, Discuss. Faraday Soc. 25, 29 (1958).

${ }^{55}$ E. L. Wee and W. G. Miller, J. Phys. Chem. 75, 1446 (1971).

${ }^{56}$ P. S. Russo and W. G. Miller, Macromolecules 16, 1690 (1983).

${ }^{57}$ F. Livolant and Y. Bouligand, J. Phys. (France) 47, 1813 (1986).

${ }^{58}$ A. Hill and A. M. Donald, Liq. Cryst. 6, 93 (1989).

${ }^{59}$ J. C. Horton, A. M. Donald, and A. Hill, Nature (London) 346, 44 (1990).

${ }^{60}$ H. Block and C. P. Shaw, Polymer 33, 2459 (1992).

${ }^{61}$ S. M. Yu, V. Conticello, G. Zhang, C. Kayser, M. J. Fournier, T. L. Mason, and D. A. Tirrell, Nature (London) 389, 167 (1997).

${ }^{62}$ D. K. Schwartz, M. L. Schlossman, and P. S. Pershan, J. Chem. Phys. 96, 2356 (1992).

${ }^{63}$ W. J. Foster, M. C. Shih, and P. S. Pershan, J. Chem. Phys. 105, 3307 (1996).

${ }^{64}$ The use of small amounts of trifluoroacetic acid (TFA) in the spreading solvent was necessary to dissolve monodisperse PBLG in chloroform, while the polydisperse PBLG dissolves in pure chloroform. The use of either pure chloroform or TFA-chloroform mixture as the spreading sol- 
vent for polydisperse PBLG samples did not have any noticeable effects on any of the measurements presented here. Since TFA is volatile, we expect most of it to evaporate from the monolayer surface after the spreading of the film, together with chloroform.

${ }^{65}$ P. S. Pershan and J. Als-Nielsen, Phys. Rev. Lett. 52, 759 (1984).

${ }^{66}$ S. K. Sinha, E. B. Sirota, S. Garoff, and H. B. Stanley, Phys. Rev. B 38, 2297 (1988).

${ }^{67}$ M. K. Sanyal, S. K. Sinha, K. G. Huang, and B. M. Ocko, Phys. Rev. Lett. 66, 628 (1991).

${ }^{68}$ J. Daillant, L. Bosio, B. Harzallah, and J. J. Benattar, J. Phys. II (France) 1, 149 (1991).

${ }^{69}$ M. Fukuto, R. K. Heilmann, P. S. Pershan, J. A. Griffiths, S. M. Yu, and D. A. Tirrell, Phys. Rev. Lett. 81, 3455 (1998).

${ }^{70}$ H. Tostmann, E. DiMasi, P. S. Pershan, B. M. Ocko, O. G. Shpyrko, and M. Deutsch, Phys. Rev. B 59, 783 (1999).

${ }^{71}$ When the singularity in $(d \sigma / d \Omega)_{\mathrm{hmg}} \sim 1 / q_{x y}^{\prime 2-\eta}$ at $q_{x y}^{\prime}=0$ falls within the detector opening $\delta q_{x} \times \delta q_{y}$, the convolution of $1 / q_{x y}^{\prime 2-\eta}$ with the rectangular resolution function $\Xi_{q}$ in Eq. (10b) can be done more conveniently by using the cylindrical coordinates $\left(q_{x y}^{\prime}, \phi^{\prime}\right)$, where $\tan \left(\phi^{\prime}\right)=q_{y}^{\prime} / q_{x}^{\prime}$.
Since $\eta>0$, the radial $q_{x y}^{\prime}$-integration at fixed $\phi^{\prime}$ analytically removes the singularity, and the azimuthal $\phi^{\prime}$-integrations can be done numerically.

${ }^{72}$ For example, Hickel et al. [Langmuir 6, 1403 (1990)] studied optical anisotropy in structurally ordered Langmuir Blodgett multilayer films formed by a different kind of $\alpha$-helical polypeptide and found the refractive index parallel to molecular axes to be only about $1 \%-1.5 \%$ higher than the indices in the perpendicular directions.

${ }^{73}$ D. K. Schwartz, M. L. Schlossman, E. H. Kawamoto, G. J. Kellogg, P. S. Pershan, and B. M. Ocko, Phys. Rev. A 41, 5687 (1990).

${ }^{74}$ J. Daillant, L. Bosio, J. J. Benattar, and J. Meunier, Europhys. Lett. 8, 453 (1989).

${ }^{75}$ If the orientational correlation length were long and the illuminated sample area viewed by the detector were not large enough to contain many randomly oriented domains, a full powder average would not be achieved. In such a case, the diffraction condition would depend on the orientation of the sample relative to the incident beam (as in the case of single crystals) and variations in peak intensity would be observed upon rotation about the surface normal. 